\title{
Floer homology of families I
}

\author{
Michael Hutchings
}

\begin{abstract}
In principle, Floer theory can be extended to define homotopy invariants of families of equivalent objects (eg Hamiltonian isotopic symplectomorphisms, 3-manifolds, Legendrian knots, etc.) parametrized by a smooth manifold $B$. The invariant of a family consists of a filtered chain homotopy type, which gives rise to a spectral sequence whose $E^{2}$ term is the homology of $B$ with local coefficients in the Floer homology of the fibers. This filtered chain homotopy type also gives rise to a "family Floer homology" to which the spectral sequence converges. For any particular version of Floer theory, some analysis needs to be carried out in order to turn this principle into a theorem. This paper constructs the invariant in detail for the model case of finite dimensional Morse homology, and shows that it recovers the Leray-Serre spectral sequence of a smooth fiber bundle. We also generalize from Morse homology to Novikov homology, which involves some additional subtleties.
\end{abstract}

$57 \mathrm{R} 58$

Floer theory is a certain kind of generalization of Morse theory, of which there are now a number of different flavors. These give invariants of symplectomorphisms, 3-manifolds, Legendrian knots, and many other types of objects. This paper describes a fundamental structure which apparently exists in most or all versions of Floer theory. The structure in question is a homotopy invariant of families of equivalent objects parametrized by a smooth manifold $B$. Its different manifestations thus give invariants of families of Hamiltonian isotopic symplectomorphisms, families of 3-manifolds, etc. The invariant of a family consists of a filtered chain homotopy type, which gives rise to a spectral sequence whose $E^{2}$ term is the homology of $B$ with local coefficients in the Floer homology of the fibers. This filtered chain homotopy type also gives rise to a "family Floer homology" to which the spectral sequence converges.

The general properties of this family invariant are stated in the "Main Principle" below. This principle cannot be formulated as a general theorem, because there is no precise definition of "Floer theory" that encompasses all of its diverse variants. For any particular version of Floer theory, in order to turn the principle into a theorem, one needs to slightly extend the construction of the Floer theory in question and check that the requisite analysis goes through. The rest of this paper constructs the invariant in detail for the model version of Floer theory, namely finite-dimensional Morse homology, 
in language designed to carry over to other versions of Floer theory. In this model situation, a family consists a smooth fiber bundle whose fibers are closed manifolds, with a family of (generically Morse) functions of the fibers. Here it turns out that the family invariant recovers the Leray-Serre spectral sequence of the fiber bundle.

The outline of this paper is as follows. The Main Principle is enunciated in Section 1. Section 2 reviews some aspects of the Morse complex that will be needed here. The spectral sequence and family Floer homology for finite dimensional Morse theory are constructed in Section 3, and some easier formal properties are established in Section 4. Section 5 identifies the spectral sequence for finite dimensional Morse theory with the Leray-Serre spectral sequence. Section 6 gives a simpler construction of the spectral sequence and family Floer homology when the base $B$ of the family is a closed manifold. In Section 7, this alternate construction is used to prove a Poincaré duality property. Section 8 explains how to refine the invariant to a filtered chain homotopy type. Section 9 generalizes from Morse homology to Novikov homology; this introduces some additional subtleties. Section 10 proves a transversality lemma which is needed in several places.

It is hoped that this paper will provide a useful reference for the construction of Floertheoretic invariants of families, by giving a detailed and systematic development in the case of Morse homology and Novikov homology. Some related ideas have appeared elsewhere, for example in Bourgeois [7], Fukaya [16], Fukaya, Seidel and Smith [17], Oancea [30], Savelyev [36], Seidel [39] and Viterbo [40]. We plan to discuss the invariant for families of symplectomorphisms in a sequel [21].

Acknowledgments It is a pleasure to thank O Buse, K Fukaya, T Kálmán, J Kedra, D McDuff, A Oancea, and P Seidel for helpful conversations and encouragement. Parts of this work were completed during visits to the MPIfM Bonn and the ETH Zürich. We thank an anonymous referee for suggesting significant improvements to the previous version of this paper.

\section{Introduction}

\subsection{Floer theory}

Roughly speaking, each version of Floer theory considered here includes the following general features. (For now we restrict attention to versions of Floer theory that are defined without using a Novikov ring. The discussion below needs to be modified slightly in the Novikov case; see Remark 1.14.) 
First, there is a topological space $S$ of "objects" whose Floer homology we will define, together with an equivalence relation on $S$, such that each equivalence class is path connected. There is also a fiber bundle $\pi: \widetilde{S} \rightarrow S$, whose fibers are contractible and represent some auxiliary choices needed to define Floer homology.

Second, for generic $X$ in a given equivalence class in $S$, and for generic $\tilde{X} \in \pi^{-1}(X)$, there is defined a free chain complex $C F_{*}(\tilde{X})$. Such an $X$ will be called "nondegenerate", and such an $\tilde{X}$ will be called "regular". The chain complex $C F_{*}$ is canonically $\mathbb{Z} / 2$-graded, and in some cases this grading can be refined to a $\mathbb{Z} / N$-grading, where $N$ is an even integer, or to a $\mathbb{Z}$-grading. The homology of this chain complex is the Floer homology, which we denote by $H F_{*}(\tilde{X})$.

To determine the signs in the differential on $C F_{*}(\tilde{X})$, one needs to choose a "coherent orientation", which is determined by an orientation choice $\mathfrak{o}_{p}$ for each generator $p$ of the chain complex. (Some global orientation choices might also be needed to define the theory.) Switching the orientation $\mathfrak{o}_{p}$ for a single generator $p$ changes the sign of exactly those differential coefficients that involve $p$. It follows that the homology does not depend on the choice of coherent orientation. Indeed, one can avoid choosing a coherent orientation by redefining the chain complex to be generated by pairs $\left(p, \mathfrak{o}_{p}\right)$, modulo the relation $\left(p,-\mathfrak{o}_{p}\right)=-\left(p, \mathfrak{o}_{p}\right)$.

Next let $X_{0}$ and $X_{1}$ be equivalent nondegenerate objects in $S$, let $\tilde{X}_{i} \in \pi^{-1}\left(X_{i}\right)$ be regular, and let $\gamma=\left\{X_{t} \mid t \in[0,1]\right\}$ be any path of equivalent objects in $S$ from $X_{0}$ to $X_{1}$. Then a generic lift of $\gamma$ to a path $\tilde{\gamma}$ in $\widetilde{S}$ from $\tilde{X}_{0}$ to $\tilde{X}_{1}$ induces a chain map

$$
\Phi(\tilde{\gamma}): C F_{*}\left(\tilde{X}_{0}\right) \longrightarrow C F_{*}\left(\tilde{X}_{1}\right),
$$

called the "continuation" map, which has the following properties:

(i) (Homotopy) A generic homotopy rel endpoints between two paths $\tilde{\gamma}_{0}$ and $\tilde{\gamma}_{1}$ with associated chain maps $\Phi_{0}$ and $\Phi_{1}$ induces a chain homotopy

$$
\begin{gathered}
K: C F_{*}\left(\tilde{X}_{0}\right) \longrightarrow C F_{*+1}\left(\tilde{X}_{1}\right), \\
\partial K+K \partial=\Phi_{0}-\Phi_{1} .
\end{gathered}
$$

(ii) (Concatenation) If the final endpoint of $\tilde{\gamma}_{1}$ is the initial endpoint of $\tilde{\gamma}_{2}$, then $\Phi\left(\tilde{\gamma}_{2} \tilde{\gamma}_{1}\right)$ is chain homotopic to $\Phi\left(\tilde{\gamma}_{2}\right) \Phi\left(\tilde{\gamma}_{1}\right)$.

(iii) (Constant) If $\tilde{\gamma}$ is a constant path then $\Phi(\tilde{\gamma})$ is the identity on chains.

These three properties imply that if $X_{0}$ and $X_{1}$ are equivalent, then there is an isomorphism $H F_{*}\left(\tilde{X}_{0}\right) \simeq H F_{*}\left(\widetilde{X}_{1}\right)$. This isomorphism is generally not canonical, because different homotopy classes of paths may induce different continuation isomorphisms on Floer homology; see Example 1.8 below. However, since $\pi^{-1}(X)$ is contractible, 
we do know that $H F_{*}(\tilde{X})$ depends only on $X$, so we denote this from now on by $H F_{*}(X)$. Moreover, if equivalence classes in $S$ are locally contractible, then the Floer homology $H F_{*}(X)$ is also defined when $X$ is degenerate, because the Floer homologies for nondegenerate objects in a contractible neighborhood $U$ of $X$ in $[X]$ are canonically isomorphic to each other via continuation along paths in $U$.

In general, one can go further in the above discussion to define "higher continuation maps". Considering property (i) again, a generic homotopy between two generic homotopies, with chain homotopies $K_{0}$ and $K_{1}$, induces a map

$$
\begin{gathered}
L: C F_{*}\left(\tilde{X}_{0}\right) \longrightarrow C F_{*+2}\left(\tilde{X}_{1}\right), \\
\partial L-L \partial=K_{0}-K_{1} .
\end{gathered}
$$

Likewise, a generic homotopy of homotopies of homotopies induces a degree three map, and so on. The purpose of this paper is to systematically exploit this sort of information to obtain Floer-theoretic invariants of families.

But returning first to our review of the standard story, the Floer homology $H F_{*}$ has three classic uses. First, for nondegenerate $X$, it gives a lower bound on the number of chain complex generators, which are sometimes geometric objects of interest. This bound is often stronger than the bound given by a signed count of the generators, which is merely the Euler characteristic $\chi\left(H F_{*}\right)$. Second, the isomorphism class of $H F_{*}$ is an invariant which can sometimes detect when two objects $X, X^{\prime}$ are not equivalent. Third, some versions of Floer theory fit into $n$-dimensional field theories as the vector spaces associated to $(n-1)$-dimensional manifolds.

Example 1.1 The prototype of Floer theory is finite dimensional Morse homology. Fix a closed smooth manifold $M$. We take $S$ to be the space of smooth functions $f: M \rightarrow \mathbb{R}$, where all functions $f$ are declared equivalent, and $\widetilde{S}$ to be the space of pairs $(f, g)$ where $g$ is a Riemannian metric on $M$. The function $f$ is nondegenerate if it is Morse, in which case the pair $(f, g)$ is regular if the $g$-gradient of $f$ satisfies the Morse-Smale transversality condition. One then defines a $\mathbb{Z}$-graded chain complex, the Morse complex, whose chains are generated by the critical points of $f$, and whose differential counts gradient flow lines between critical points. The homology of this complex is canonically isomorphic to the ordinary singular homology of $M$. For more details see eg Austin and Braam [1], Bott [6], Salamon [35], Schwarz [37] and Witten [41] and the review in Section 2.

Example 1.2 Given a (generic) symplectomorphism $\phi$ of a closed symplectic manifold $(M, \omega)$, one can define a chain complex whose chains are generated by the (nondegenerate) fixed points of $\phi$, and whose differential counts certain pseudoholomorphic 
cylinders in $\mathbb{R}$ cross the mapping torus of $\phi$. The differential in the chain complex depends on the auxiliary choice of a generic one-parameter family of $\omega$-tame almost complex structures $J_{t}$ on $M$ with $J_{t+1}=\phi_{*}^{-1} \circ J_{t} \circ \phi_{*}$ (and in general on some abstract perturbations needed to obtain transversality). The homology of the complex depends only on the (weakly) Hamiltonian isotopy class of $\phi$. This theory has many applications, for example to prove the Arnold conjecture (see eg Salamon [35]), and to detect pairs of symplectomorphisms which are smoothly but not symplectically isotopic to each other (see Seidel [38]).

Example 1.3 Seiberg-Witten Floer theory (and the conjecturally equivalent Heegaard Floer theory of Ozsváth-Szabó [32]) associates a package of Floer homology groups to a pair $(Y, \mathfrak{s})$, where $Y$ is a closed oriented 3 -manifold and $\mathfrak{s}$ is a spin-c structure on $Y$; see Kronheimer and Mrowka [25] and Kronheimer, Mrowka, Ozsváth and Szabó [26]. A compact four-manifold with boundary determines relative invariants living in the Floer homology groups of the boundary, and these enter into product formulas for the Seiberg-Witten invariants of closed four-manifolds cut along three-manifolds.

Example 1.4 The relative contact homology of Chekanov and Eliashberg-Hofer [8; 13] associates to a (generic) Legendrian knot in $\mathbb{R}^{3}$ a differential graded algebra whose chains are generated by words in the crossings of the $x-y$ projection of the knot, and whose differential counts certain holomorphic discs which can be understood combinatorially in terms of polygons in the $x-y$ projection. The homology of this DGA is an invariant of Legendrian isotopy, and has been used in [8] to distinguish Legendrian knots whose classical invariants agree. This theory is vastly generalized to contact manifolds with or without Legendrian submanifolds in the contact homology and symplectic field theory of Eliashberg-Givental-Hofer [12].

\subsection{Invariants of families}

We now consider the Floer homology of a family of equivalent objects in $S$, parametrized by a (finite dimensional) smooth manifold $B$.

The appropriate notion of "family" depends on the version of Floer theory under consideration. In any version of Floer theory, one can obtain a family as a map from $B$ to an equivalence class in $S$. However, it is sometimes more interesting to consider appropriate "fiber bundles" over $B$ whose fibers are equivalent elements of $S$. In Example 1.1 above, we consider a smooth fiber bundle $Z \rightarrow B$, where the fibers are finite dimensional closed manifolds, together with a smooth function $f: Z \rightarrow \mathbb{R}$. In Example 1.2, it is already interesting to consider a map to the symplectomorphism 
group, $B \rightarrow \operatorname{Symp}(M, \omega)$, whose image consists of weakly Hamiltonian isotopic symplectomorphisms. More generally, one can consider a symplectic fibration together with a symplectomorphism of each fiber satisfying a weak Hamiltonian isotopy condition. In Example 1.3, a family consists of a smooth fiber bundle $Z \rightarrow B$ whose fibers are closed oriented 3-manifolds, together with a fiberwise spin-c structure, ie a lifting of the fiberwise frame bundle from $\mathrm{SO}(3)$ to $\operatorname{Spin}^{c}(3) \simeq \mathrm{U}(2)$. In Example 1.4, one can start by considering a map from $B$ to $\operatorname{Leg}\left(S^{1}, \mathbb{R}^{3}\right)$, the space of all Legendrian embeddings of $S^{1}$ into $\mathbb{R}^{3}$.

In general, if the family $Z$ over $B$ is generic, then for $b \in B$ in the complement of a codimension 1 subvariety, the object $Z_{b} \in S$ is nondegenerate so that the Floer homology $H F_{*}\left(Z_{b}\right)$ is defined. Such a family will be called "admissible". Also, one can extend the continuation map (1-1) to families over $[0,1]$ in order to show that for generic $b_{0}, b_{1} \in B$, a path from $b_{0}$ to $b_{1}$ induces an isomorphism between the Floer homologies over $b_{0}$ and $b_{1}$, satisfying the homotopy properties (i), (ii), and (iii) of Section 1.1. These continuation isomorphisms assemble the fiberwise Floer homologies $H F_{*}\left(Z_{b}\right)$ into a locally constant sheaf, or local coefficient system, defined over all of $B$, which we denote by $\mathcal{F}_{*}(Z)$. Note that the Floer homology over $b \in B$ is well-defined even when $Z_{b}$ is degenerate, because the Floer homologies over all generic $b^{\prime}$ in a contractible neighborhood $U$ of $b$ in $B$ are canonically isomorphic to each other via continuation along paths in $U$.

As before, the fiberwise Floer homology $\mathcal{F}_{*}(Z)$ is canonically $\mathbb{Z} / 2$-graded, and it is sometimes possible to refine the grading of an individual fiber. However, when this refinement is not canonical and $B$ is not simply connected, there might be obstructions to refining the grading continuously for all the fibers in the family.

Main Principle For a version of Floer theory as above, let $Z$ be an admissible family of equivalent objects in $S$ parametrized by a smooth manifold $B$. Then there exists a spectral sequence $E_{*, *}^{*}$, defined from $E^{2}$ on, satisfying properties (a) through (g) below:

(a) ( $E^{2}$ term) The $E^{2}$ term is given by the homology with local coefficients

$$
E_{i, j}^{2}=H_{i}\left(B ; \mathcal{F}_{j}(Z)\right) .
$$

(b) (Homotopy invariance) Suppose that $Z$ is an admissible family over $[0,1] \times B$, such that the restrictions $Z_{0}:=\left.Z\right|_{\{0\} \times B}$ and $Z_{1}:=\left.Z\right|_{\{1\} \times B}$ are admissible. Then there is an isomorphism of spectral sequences

$$
E_{*, *}^{*}\left(Z_{0}\right) \simeq E_{*, *}^{*}\left(Z_{1}\right) .
$$


On the $E^{2}$ terms, this is the isomorphism

$$
H_{i}\left(B ; \mathcal{F}_{j}\left(Z_{0}\right)\right) \simeq H_{i}\left(B ; \mathcal{F}_{j}\left(Z_{1}\right)\right)
$$

induced by the isomorphism of local coefficient systems $\mathcal{F}_{j}\left(Z_{0}\right) \simeq \mathcal{F}_{j}\left(Z_{1}\right)$ defined by continuation along paths $[0,1] \times\{b\}$ for $b \in B$.

(c) (Naturality) If $\phi: B^{\prime} \rightarrow B$ is generic so that the family $\phi^{*} Z$ over $B^{\prime}$ is admissible, then the pushforward in homology

$$
\phi_{*}: H_{*}\left(B^{\prime} ; \mathcal{F}_{*}\left(\phi^{*} Z\right)\right) \longrightarrow H_{*}\left(B ; \mathcal{F}_{*}(Z)\right)
$$

extends to a morphism of spectral sequences

$$
\phi_{*}: E_{*, *}^{*}\left(\phi^{*} Z\right) \longrightarrow E_{*, *}^{*}(Z) .
$$

(d) (Triviality) If $Z=B \times X$ is a constant family with $X$ nondegenerate, then the spectral sequence collapses at $E^{2}$.

Remark 1.5 Property (b) follows from properties (a) and (c). (Proof: By property (c), the two inclusions $B \rightarrow B \times[0,1]$ sending $x \in B$ to $(x, 0)$ and $(x, 1)$ respectively induce morphisms of spectral sequences from $E^{2}$ on. By property (a) and Equation (1-6), these morphisms restrict to isomorphisms on $E^{2}$, hence on all higher terms as well; see Remark 4.2.) Also, (1-6) implies that the maps (1-7) are functorial and homotopy invariant. When equivalence classes in $S$ are locally contractible, the homotopy invariance property (b) implies that the spectral sequence is well-defined and has the above properties even for non-admissible families. There is also a variant of property (d) (see Proposition 4.6) which gives an obstruction to obtaining regularity of all fibers of a family.

The next property in the Main Principle involves duality. For any notion of Floer homology, there is a dual notion of Floer cohomology obtained by dualizing the chain complex. Likewise, one can algebraically dualize the construction of the spectral sequence to obtain a cohomological spectral sequence $E_{*}^{*, *}$ with $E_{2}^{i, j}=H^{i}\left(B ; \mathcal{F}^{j}(Z)\right)$, which satisfies dual versions of the properties above. Some versions of Floer theory also admit a more nontrivial duality, where for each nondegenerate object $X \in S$ there is a nondegenerate "dual object" $X^{\vee}$ satisfying a "Poincaré duality"

$$
H F_{*}\left(X^{\vee}\right)=H F^{-*}(X),
$$

up to an even grading shift (which is implicit below). For example, in finite dimensional Morse theory of closed oriented manifolds, one replaces the Morse function $f$ with $-f$; in Floer theory of symplectomorphisms one replaces $\phi$ with $\phi^{-1}$; in Seiberg-Witten 
Floer theory one switches the orientation of the three-manifold. In such a version of Floer theory, a family $Z$ has a dual family $Z^{\vee}$ obtained by replacing each fiber with its dual. If $B$ is closed and oriented, then by property (a), Poincaré duality on $B$ with local coefficients gives an isomorphism

$$
E_{i, j}^{2}\left(Z^{\vee}\right)=E_{2}^{\operatorname{dim}(B)-i,-j}(Z) .
$$

(e) (Poincaré duality) For any version of Floer theory admitting a duality as above, if $B$ is closed and oriented, then the isomorphism (1-9) extends to a canonical isomorphism of spectral sequences

$$
E_{*, *}^{*}\left(Z^{\vee}\right)=E_{*}^{\operatorname{dim}(B)-*,-*}(Z) .
$$

With more work, the spectral sequence invariant can be refined slightly to a "filtered chain homotopy type". More precisely:

Definition 1.6 Let $C_{*}$ be a chain complex with an increasing filtration $F_{i} C_{*} \subset$ $F_{i+1} C_{*}$, and let $C_{*}^{\prime}$ be another such filtered chain complex. A chain map $\phi: C_{*} \rightarrow C_{*}^{\prime}$ is a filtered chain map if $\phi\left(F_{i} C_{*}\right) \subset F_{i} C_{*}^{\prime}$. A filtered chain homotopy between two filtered chain maps $\phi_{0}, \phi_{1}: C_{*} \rightarrow C_{*}^{\prime}$ is a module homomorphism $K: C_{*} \rightarrow C_{*+1}^{\prime}$ such that $K\left(F_{i} C_{*}\right) \subset F_{i+1} C_{*+1}$ and $\partial K+K \partial=\phi_{0}-\phi_{1}$. A filtered chain map $\phi: C_{*} \rightarrow C_{*}^{\prime}$ is a filtered chain homotopy equivalence if there is a filtered chain map $\phi^{\prime}: C_{*}^{\prime} \rightarrow C_{*}$ such that $\phi^{\prime} \circ \phi$ and $\phi \circ \phi^{\prime}$ are filtered chain homotopic to the identity on $C_{*}$ and $C_{*}^{\prime}$ respectively. This defines an equivalence relation on filtered chain complexes. A filtered chain homotopy type is a filtered chain homotopy equivalence class of filtered chain complexes.

Note that contrary to what one might expect, our "filtered chain homotopies" are allowed to increase the filtration by 1 . Nonetheless, a filtered chain homotopy type in the above sense has a well-defined, filtered, homology, and determines a spectral sequence which is defined from the $E^{2}$ term on.

We can now append the following properties to the Main Principle:

(f) There is a filtered chain complex $\mathfrak{C}_{*}(Z)$, which induces the spectral sequence $E_{*, *}^{*}(Z)$, and whose filtered chain homotopy type is homotopy invariant and natural in the following sense:

$\left(b^{\prime}\right)$ Under the assumptions of (b), there is a filtered chain homotopy equivalence

$$
\Phi: \mathfrak{C}_{*}\left(Z_{0}\right) \longrightarrow \mathfrak{C}_{*}\left(Z_{1}\right),
$$

which induces the isomorphism on spectral sequences (1-5). 
$\left(c^{\prime}\right)$ Under the assumptions of (c), the morphism of spectral sequences (1-7) is induced by a filtered chain map

$$
\phi_{*}: \mathfrak{C}_{*}\left(\phi^{*} Z\right) \longrightarrow \mathfrak{C}_{*}(Z) .
$$

Statement $\left(\mathrm{b}^{\prime}\right)$ also has a refined version, asserting that the filtered chain homotopy equivalences (1-11) have formal properties analogous to those of continuation isomorphisms. Statement $\left(\mathrm{c}^{\prime}\right)$ also has a refined version, asserting that the filtered chain maps (1-12) are functorial, and homotopy invariant (up to filtered chain homotopy) with respect to the isomorphisms in $\left(\mathrm{b}^{\prime}\right)$. For the precise statements see Propositions 8.6 and 8.7.

We call the homology of $\mathfrak{C}_{*}(Z)$ the family Floer homology, and denote it by $H F_{*}(Z)$. This is a filtered module, whose associated graded is determined from the spectral sequence $E_{*, *}^{*}(Z)$ by

$$
G_{i} H F_{j}(Z)=E_{i, j-i}^{\infty}(Z)=E_{i, j-i}^{\operatorname{dim}(B)+1}(Z) .
$$

The family Floer homology has the following additional properties. To state the first property, let $\mathfrak{C}^{*}$ denote the dual of $\mathfrak{C}_{*}$, and define the family Floer cohomology $H F^{*}(Z)$ to be the homology of $\mathfrak{C}^{*}$. The increasing filtration on $\mathfrak{C}_{*}$ induces a decreasing filtration on $\mathfrak{C}^{*}$, namely $F^{i} \mathfrak{C}^{*}:=\operatorname{Ann}\left(F_{i-1} \mathfrak{C}_{*}\right)$, and this defines a decreasing filtration on $H F^{*}(Z)$.

$\left(\mathrm{e}^{\prime}\right)$ Under the assumptions of (e), there is a canonical isomorphism

$$
F_{i} H F_{j}(Z)=F^{\operatorname{dim}(B)-i} H F^{\operatorname{dim}(B)-j}\left(Z^{\vee}\right) .
$$

(g) (Mayer-Vietoris) If $U$ and $V$ are open sets in $B$, then there is a long exact sequence of filtered modules

$$
\begin{aligned}
\longrightarrow H F_{*}\left(\left.Z\right|_{U \cap V}\right) \longrightarrow H F_{*}\left(\left.Z\right|_{U}\right) \oplus H F_{*}\left(\left.Z\right|_{V}\right) \longrightarrow & H F_{*}\left(\left.Z\right|_{U \cup V}\right) \\
& \longrightarrow H F_{*-1}\left(\left.Z\right|_{U \cap V}\right) \longrightarrow .
\end{aligned}
$$

This concludes the statement of the Main Principle. By itself the statement is somewhat vacuous, in that one could define the family invariants in a trivial manner with the spectral sequence always collapsing at $E^{2}$ and so forth. The point is that there is a natural way to construct the family invariants, which involves a slight extension of standard constructions in Floer theory, and which turns out to be nontrivial.

In fact, we will give two different constructions of the spectral sequence and family Floer homology. The first construction couples the Floer homology of the fibers to cubical singular homology on $B$, and is useful for proving formal properties. When $B$ 
is a closed manifold, there is a second, equivalent construction using Morse homology on $B$, which is more practical for direct computations.

\subsection{Examples}

Example 1.7 Theorem 5.1 below shows that the spectral sequence for finite dimensional Morse theory recovers the Leray-Serre spectral sequence of a smooth fiber bundle whose fibers are closed manifolds. In this case the family Floer homology is just the singular homology of the total space.

Example 1.8 (Monodromy) For any version of Floer theory as above, given a family over $S^{1}$ with a (nondegenerate) fiber $X$, continuation around the circle induces a monodromy map $\Phi_{*}: H F_{*}(X) \rightarrow H F_{*}(X)$. This information is fed into the spectral sequence in the definition of the $E^{2}$ term: $E_{0, j}^{2} \simeq H F_{j}(X) / \operatorname{Im}\left(1-\Phi_{*}\right)$ and $E_{1, j}^{2}=$ $\operatorname{Ker}\left(1-\Phi_{*}\right) \subset H F_{j}(X)$. This monodromy defines a multiplicative homomorphism

$$
\pi_{1}([X], X) \longrightarrow \operatorname{Aut}\left(H F_{*}(X)\right) .
$$

Seidel [39] introduced a version of this homomorphism for Floer homology of Hamiltonian symplectomorphisms of a fixed symplectic manifold, and obtained applications to $\pi_{1}$ of Hamiltonian symplectomorphism groups and relations with quantum cohomology. (Seidel's homomorphism depends on an additional choice, because it uses Floer homology with Novikov rings; see Remark 1.14 and Example 9.12. Also, the definition of this map in [39] is not stated in terms of continuation maps, but is easily seen to be equivalent to this.)

Bourgeois [7] used this monodromy for contact homology of contact manifolds to detect an infinite cyclic subgroup of $\pi_{1}$ of each component of the space of tight contact structures on $T^{3}$. This reproved a result of Geiges and Gonzalo [18].

For contact homology of Legendrian knots in $\mathbb{R}^{3}$, Kálmán [24] constructed a combinatorial version of this monodromy (which is equivalent to the monodromy defined analytically by counting holomorphic discs [11]), and used it to detect a homotopically nontrivial one-parameter family of Legendrian knots in $\mathbb{R}^{3}$ which is nullhomotopic in the space of smooth embeddings.

Example 1.9 (Homotopy groups) Suppose $B=S^{k}$ with $k>1$. Let $X \in S$ be a (nondegenerate) object in the equivalence class $[X]$. Consider a family given by a map $S^{k} \longrightarrow[X]$ sending a distinguished point on $S^{k}$ to $X$. In this case the content of the spectral sequence is the $k^{\text {th }}$ differential, which is a map

$$
\delta_{k}: H F_{*}(X) \longrightarrow H F_{*+k-1}(X) .
$$


By homotopy invariance (b), we obtain a well-defined map

$$
\pi_{k}([X], X) \longrightarrow \operatorname{End}_{k-1}\left(H F_{*}(X)\right) .
$$

Furthermore, the map (1-14) is an additive homomorphism. One can see this by applying naturality (c) to three maps $S^{k} \longrightarrow N\left(S^{k} \bigvee S^{k}\right)$, namely the two inclusions and the map that pinches the equator to a point. Here $N$ denotes some thickening of $S^{k} \bigvee S^{k}$ to a manifold that retracts onto it. One can also see additivity more directly from the following alternate description of $\delta_{k}$.

Remark 1.10 (Higher continuation maps) For this special case of $B=S^{k}$, if we identify $S^{k}=I^{k} / \partial I^{k}$, then the differential (1-13) is induced by the higher continuation maps of Section 1.1. For example, if $k=2$, then pulling back our family to $I^{2}$ gives a homotopy from a constant path in $S$ to itself. Thus the chain homotopy $K$ in (1-2) is a degree 1 chain map, and the map $L$ in (1-3) shows that the induced map on homology $K_{*}$ is homotopy invariant. Remark 6.2 explains why $\delta_{2}=K_{*}$.

Example 1.11 (Hamiltonian symplectomorphism groups) In [21], we specialize Example 1.9 above to Floer theory of symplectomorphisms of a closed symplectic manifold $(M, \omega)$, under some monotonicity assumptions, to obtain a map

$$
\Psi: \pi_{k}(\operatorname{Ham}(M, \omega), \mathrm{id}) \longrightarrow \operatorname{End}_{1-k}\left(Q H^{*}(M)\right) .
$$

Here Ham denotes the Hamiltonian symplectomorphism group, $Q H^{*}(M)$ is the quantum cohomology of $M$, and $\operatorname{End}_{1-k}$ denotes the $Q H^{*}(M)$-module endomorphisms of degree $1-k$. The invariant $\Psi$ can distinguish some homotopy classes, although in general little is known about it.

Example 1.12 Bourgeois [7] has independently constructed the invariant (1-14) as in Remark 1.10 for a version of contact homology, and applied it to detect an infinite cyclic subgroup of $\pi_{3}$ of the space of contact structures on $T^{4} \times S^{3}$, based at the standard contact structure on the unit cotangent bundle of $T^{4}$.

Example 1.13 (Relative invariants) For those versions of Floer theory that arise as recipients of relative invariants of manifolds with boundary, the family Floer homology is the natural recipient for relative invariants of families of manifolds with boundary. This will be explained in detail elsewhere.

Remark 1.14 (Novikov generalization) For versions of Floer homology defined over a Novikov ring, the above discussion needs to be modified as follows. First, the space of auxiliary choices needed to define Floer homology is no longer contractible. A related 
fact is that the continuation map (1-1) is only defined, and only satisfies properties (i)-(iii), up to multiplication by a certain group of units in the Novikov ring. As a consequence, for each family $Z$ over $B$ there is an obstruction to defining the local coefficient system $\mathcal{F}_{*}(Z)$. The obstruction lives in $H^{2}(B ; \Gamma)$, where $\Gamma$ is a certain local coefficient system on $B$. When the obstruction vanishes, there are different choices for $\mathcal{F}_{*}(Z)$ which are classified by $H^{1}(B ; \Gamma)$. Each such choice leads to a different spectral sequence, satisfying straightforward analogues of the above formal properties. The details of this are explained in Section 9.

\section{Review of the Morse complex}

We now review those aspects of the Morse complex that we will be using and generalizing in the rest of this paper.

\subsection{Definition of the Morse complex}

Let $X$ be a closed smooth manifold, let $f: X \rightarrow \mathbb{R}$ be a Morse function, and let $g$ be a metric on $X$. Let $\xi$ denote the negative gradient of $f$ with respect to $g$. If $p$ is a critical point of $f$, the descending manifold $\mathcal{D}(p)$ is the unstable manifold of $p$ for $\xi$, ie the set of all $x \in X$ such that the flow of $-\xi$ starting at $x$ converges to $p$. Likewise the ascending manifold $\mathcal{A}(p)$ is the set of $x \in X$ such that the flow of $\xi$ starting at $x$ converges to $p$. We assume that the pair $(f, g)$ is Morse-Smale, ie all ascending and descending manifolds intersect transversely. Given a Morse function $f$, this holds for generic metrics $g$.

If $p$ and $q$ are critical points, a flow line from $p$ to $q$ is a map $u$ : $\mathbb{R} \rightarrow X$ such that $u^{\prime}(s)=\xi(s), \lim _{s \rightarrow-\infty} u(s)=p$, and $\lim _{s \rightarrow+\infty} u(s)=q$. For $p \neq q$, let $\mathcal{M}(p, q)$ denote the moduli space of flow lines from $p$ to $q$, modulo the free action of $\mathbb{R}$ by precomposing with translations. The Morse-Smale condition implies that $\mathcal{M}(p, q)$ is a manifold of dimension

$$
\operatorname{dim}(\mathcal{M}(p, q))=|p|-|q|-1 .
$$

Here $|p|$ denotes the Morse index of $p$.

We choose an orientation of the descending manifold of each critical point, and we denote this collection of orientation choices by $\mathfrak{o}$. This determines orientations of the manifolds $\mathcal{M}(p, q)$; we use the convention in Salamon [35]. Note that an orientation of $X$ is not needed. 
The Morse complex $C_{*}=C_{*}^{\text {Morse }}(f, g, \mathfrak{o})$ is the following $\mathbb{Z}$-graded complex of $\mathbb{Z}$ modules. The chain module $C_{i}$ is the free $\mathbb{Z}$-module generated by the critical points of index $i$. The differential $\partial: C_{i} \rightarrow C_{i-1}$ is defined by

$$
\partial p:=\sum_{q \in \operatorname{Crit}_{i-1}(f)} \# \mathcal{M}(p, q) \cdot q
$$

where $p$ is a critical point of index $i$ and $\mathrm{Crit}_{i-1}(f)$ denotes the set of index $i-1$ critical points of $f$. Here "\#” denotes the number of points counted with signs as in the previous paragraph.

Standard compactness and gluing arguments (see eg Austin and Braam [1], Barraud and Cornea [2] and Cohen, Jones and Segal [9]) show that the moduli space $\mathcal{M}(p, q)$ has a compactification to a manifold with corners $\overline{\mathcal{M}}(p, q)$, whose codimension $k$ stratum consists of " $k$-times broken flow lines":

$$
\overline{\mathcal{M}}(p, q)_{k}=\coprod_{p=r_{0}, r_{1}, \ldots, r_{k}, r_{k+1}=q \text { distinct }} \mathcal{M}\left(r_{0}, r_{1}\right) \times \cdots \times \mathcal{M}\left(r_{k}, r_{k+1}\right) .
$$

In particular the boundary, as an oriented topological manifold, is given by

$$
\partial \overline{\mathcal{M}}(p, q)=\bigcup_{r}(-1)^{|p|-|r|-1} \overline{\mathcal{M}}(p, r) \times \overline{\mathcal{M}}(r, q) .
$$

It follows that $\partial$ is well-defined and $\partial^{2}=0$. We denote the homology of this chain complex by $H_{*}^{\text {Morse }}(f, g, \mathfrak{o})$.

Henceforth, we will usually suppress $\mathfrak{o}$ from the notation, because as in Section 1.1, the Morse homology does not depend on $\mathfrak{o}$; and one can also rephrase the definition so that no orientation choices are made in the first place.

\subsection{Continuation maps}

Let $\left(f_{0}, g_{0}\right)$ and $\left(f_{1}, g_{1}\right)$ be Morse-Smale pairs on $X$. Given any smooth path $\left\{f_{t} \mid t \in[0,1]\right\}$ from $f_{0}$ to $f_{1}$, if one subsequently chooses a generic smooth path $\left\{g_{t} \mid t \in[0,1]\right\}$ from $g_{0}$ to $g_{1}$, then these induce a continuation map

$$
\Phi\left(\left\{\left(f_{t}, g_{t}\right)\right\}\right): C_{*}^{\text {Morse }}\left(f_{0}, g_{0}\right) \longrightarrow C_{*}^{\text {Morse }}\left(f_{1}, g_{1}\right),
$$

which can be defined as follows. Fix once and for all a smooth function $\beta:[0,1] \rightarrow \mathbb{R}$ such that $\beta(t) \geq 0, \beta^{-1}(0)=\{0,1\}, \beta^{\prime}(0)>0$, and $\beta^{\prime}(1)<0$; different choices of $\beta$ will give rise to chain homotopic continuation maps. Now $\Phi$ is a signed count of flow lines of the vector field

$$
\beta(t) \partial_{t}+\xi_{t}
$$


on $[0,1] \times X$, from critical points of $f_{0}$ to critical points of $f_{1}$. Here $t$ denotes the $[0,1]$ coordinate. (Usually continuation maps are defined by counting flow lines of a vector field on $\mathbb{R} \times X$ instead of $[0,1] \times X$. The definition of continuation maps given here is related to a special case of the notion of "Morse cobordism" studied in [10].)

This $\Phi$ is a chain map and satisfies the homotopy properties (i), (ii), and (iii) listed in Section 1.1, thus inducing an isomorphism

$$
\Phi\left(\left\{\left(f_{t}, g_{t}\right)\right\}\right)_{*}: H_{*}^{\text {Morse }}\left(f_{0}, g_{0}\right) \stackrel{\simeq}{\longrightarrow} H_{*}^{\text {Morse }}\left(f_{1}, g_{1}\right) .
$$

Note in particular that the chain homotopy $K$ associated to a homotopy $\left\{\left(f_{s, t}, g_{s, t}\right) \mid\right.$ $s, t \in[0,1]\}$ counts flow lines of the vector field

$$
\beta(t) \partial_{t}+\xi_{s, t}
$$

on $[0,1]^{2} \times X$. The higher continuation maps associated to higher homotopies mentioned in Section 1.1 count flow lines of the analogous vector field on $[0,1]^{k} \times X$.

Remark 2.1 (Bifurcation analysis) Another way to obtain an isomorphism as in (2-3), which we will not use here, is to explicitly construct a chain map $\Phi^{\prime}$ by studying the bifurcations of the family $\left\{\left(f_{t}, g_{t}\right)\right\}$; see eg Floer [14], Hutchings [22] and Laudenbach [28]. The bifurcation chain map $\Phi^{\prime}$ does not always agree with the continuation chain map $\Phi$. For example, even when $\left(f_{t}, g_{t}\right)$ is Morse-Smale for all $t$, sometimes $\Phi$ is not the obvious identification of critical points. However, we conjecture that for a given family $\left\{\left(f_{t}, g_{t}\right)\right\}$, the bifurcation chain map $\Phi^{\prime}$ agrees with the continuation map $\Phi$ if one redefines $\Phi$ by replacing the function $\beta$ in Equation (2-2) by $\epsilon \beta$ for $\epsilon>0$ sufficiently small.

\subsection{The isomorphism with singular homology}

There is a canonical isomorphism from Morse homology to singular homology, which in the notation below is

$$
\Psi_{*}: H_{*}^{\text {Morse }}(f, g) \stackrel{\simeq}{\longrightarrow} H_{*}(X) .
$$

To define this, first note that the descending manifold $\mathcal{D}(p)$ has a compactification to a manifold with corners $\overline{\mathcal{D}}(p)$ (see eg Austin and Braam [1] and Hutchings and Lee [23]) with

$$
\begin{gathered}
\overline{\mathcal{D}}(p)_{k}=\coprod_{p=r_{0}, r_{1}, \ldots, r_{k} \text { distinct }} \mathcal{M}\left(r_{0}, r_{1}\right) \times \cdots \times \mathcal{M}\left(r_{k-1}, r_{k}\right) \times \mathcal{D}\left(r_{k}\right), \\
\partial \overline{\mathcal{D}}(p)=\bigcup_{r}(-1)^{|p|-|r|-1} \overline{\mathcal{M}}(p, r) \times \overline{\mathcal{D}}(r)
\end{gathered}
$$


The inclusion $\mathcal{D}(p) \rightarrow X$ extends to a continuous "endpoint map"

$$
e: \overline{\mathcal{D}}(p) \longrightarrow X,
$$

defined by projecting onto the $\mathcal{D}\left(r_{k}\right)$ factor in (2-6).

Now one way to obtain the isomorphism (2-5) is to show that the compactified descending manifolds $\overline{\mathcal{D}}(p)$ are homeomorphic to closed balls (see eg Barraud and Cornea [2] and Biran [3]), so that together with the maps $e: \overline{\mathcal{D}}(p) \rightarrow X$, they give $X$ the structure of a CW complex in which the cellular chain complex agrees with the Morse complex. However, for this paper, we will need chain maps going directly between Morse homology and cubical singular homology, which we define as follows.

For each pair of distinct critical points $p, q$, choose a cubical singular chain $m_{p, q} \in$ $C_{|p|-|q|-1}(\overline{\mathcal{M}}(p, q))$ representing the fundamental class of the oriented topological manifold $\overline{\mathcal{M}}(p, q)$ relative to its boundary. As in [2], by Equation (2-1) we can choose the $m_{p, q}$ 's by induction on $|p|-|q|$ so that

$$
\partial m_{p, q}=\sum_{r}(-1)^{|p|-|r|-1} m_{p, r} \times m_{r, q} .
$$

Here $\times$ denotes the cross product of cubical chains. Likewise, by Equation (2-7) and induction on $|p|$, the fundamental class of $\overline{\mathcal{D}}(p)$ can be represented by a cubical singular chain $d_{p} \in C_{|p|}(\overline{\mathcal{D}}(p))$ such that

$$
\partial d_{p}=\sum_{r}(-1)^{|p|-|r|-1} m_{p, r} \times d_{r} .
$$

Finally, define $\Psi: C_{*}^{\text {Morse }}(f, g) \rightarrow C_{*}(X)$ by $\Psi(p):=e_{*}\left(d_{p}\right)$. This is a chain map by Equation (2-9). (Note that $e_{*}$ sends terms on the right hand side of (2-9) with $|p|-|r|>1$ to linear combinations of degenerate cubes, which are quotiented out in the cubical chain complex.) Furthermore, it induces an isomorphism on homology.

The inverse map on homology can be described as follows; cf [23]. The homology of $X$ can be computed by the subcomplex $C_{*}^{\prime}(X)$ of the cubical singular chain complex generated by smooth cubes $\sigma:[-1,1]^{i} \rightarrow X$ that are transverse to the ascending manifolds of the critical points. If $p$ is a critical point of index $i$, let $\mathcal{M}(\sigma, p)$ denote the moduli space of pairs $(x, u)$, where $x \in[-1,1]^{i}$ and $u:[0, \infty) \rightarrow X$ is a flow line of $\xi$ starting at $\sigma(x)$ and converging to $p$. The moduli space $\mathcal{M}(\sigma, p)$ is finite and has a natural orientation induced by $\mathfrak{o}$. The inverse of $\Psi_{*}$ is then induced by a chain map $\Xi: C_{*}^{\prime}(X) \rightarrow C_{*}^{\text {Morse }}(f, g)$ defined by

$$
\Xi(\sigma):=\sum_{p \in \mathrm{Crit}_{i}(f)} \# \mathcal{M}(\sigma, p) \cdot p .
$$


A slight modification of the construction of $\Psi$ or $\Xi$ shows that the isomorphism $\Psi_{*}$ commutes with continuation, ie we have a commutative diagram

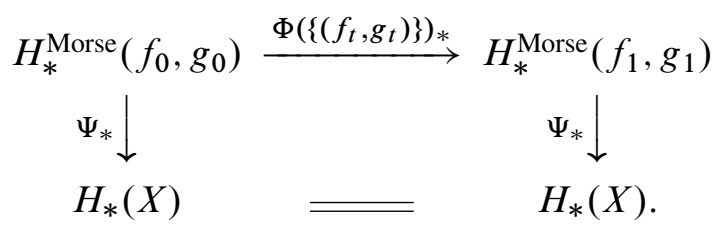

Remark 2.2 (Local coefficients) The above definitions of the Morse complex, the continuation map, and the isomorphism between Morse homology and singular homology all work just as well with coefficients in any local coefficient system on $X$. Since all of the above definitions count flow lines of vector fields, to work with local coefficients one simply needs to incorporate the "parallel transport" of the local coefficient system along these flow lines.

\section{The main construction}

We now construct the filtered chain complex for Morse homology of families.

\subsection{Families of (generically) Morse functions}

In the context of finite dimensional Morse theory, a family consists of a triple $(\pi, f, \nabla)$. Here $\pi: Z \rightarrow B$ is a smooth fiber bundle, where $B$ is any finite dimensional smooth manifold, and the fibers are finite dimensional closed manifolds. Also $f$ is a family of smooth functions $f_{b}: Z_{b} \rightarrow \mathbb{R}$ for each $b \in B$, depending smoothly on $b$. Of course the family $\left\{f_{b}\right\}$ is equivalent to a smooth function $f: Z \rightarrow \mathbb{R}$; but keeping Floer-theoretic generalizations in mind, we prefer to regard it as a fiberwise object. We assume that the family $\left\{f_{b}\right\}$ is "admissible" in the following sense:

Definition 3.1 The family $\left\{f_{b}\right\}$ is admissible if the function $f_{b}$ is Morse for $b$ in the complement of a codimension one subvariety of $B$.

Generic families are admissible in this sense. Note that where $f_{b}$ is not Morse, it is allowed to have arbitrarily bad singularities. Finally, $\nabla$ is a connection on $Z \rightarrow B$. We often denote the family $(\pi, f, \nabla)$ simply by $Z$.

In order to fix signs, for each $b \in B$ such that $f_{b}$ is Morse, we choose an orientation of the descending manifold of each critical point of $f_{b}$ in $Z_{b}$. Note that although the descending manifold depends on the choice of a metric on $Z_{b}$, for any two metrics there is a canonical identification between orientations of the corresponding descending 
manifolds. We denote this set of orientation choices by $\mathfrak{o}$. Our family is really a quadruple $(\pi, f, \mathfrak{o}, \nabla)$, but we usually suppress $\mathfrak{o}$ in the notation, for the reasons explained previously.

A path $\gamma:[0,1] \rightarrow B$ with $f_{\gamma(0)}$ and $f_{\gamma(1)}$ Morse induces an isomorphism between the corresponding Morse homologies, in which the vector field (2-2) is replaced by the vector field

$$
H\left(\beta(t) \partial_{t}\right)+\xi_{t}
$$

on the pullback bundle $\gamma^{*} Z$. Here $H$ denotes the horizontal lift with respect to the connection $\gamma^{*} \nabla$. As explained in Section 1.2, these continuation isomorphisms assemble the Morse homology of the fibers into a well-defined local coefficient system $\mathcal{F}_{*}(Z)$ on $B$. By $(2-5)$ and (2-11), we secretly know that this local coefficient system is canonically isomorphic to the homology of the fibers of $Z \rightarrow B$ :

$$
\mathcal{F}_{*}(Z)=\left\{H_{*}\left(Z_{b}\right)\right\}
$$

\subsection{Cubes and vector fields}

The differential in our filtered chain complex will count flow lines of a vector field $V$ which we now define. First, define a vector field $W_{i}$ on the $i$-cube $[-1,1]^{i}$ by

$$
W_{i}:=-\sum_{\mu=1}^{i}\left(x_{\mu}+1\right) x_{\mu}\left(x_{\mu}-1\right) \partial_{\mu} .
$$

This is the negative gradient, with respect to the Euclidean metric, of the Morse function

$$
\frac{1}{4} \sum_{\mu=1}^{i}\left(x_{\mu}+1\right)^{2}\left(x_{\mu}-1\right)^{2}
$$

on $[-1,1]^{i}$, which has a critical point of index $k$ at the center of each $k$-face. The descending manifold of such a critical point is simply the open face. We choose orientations of the faces, once and for all, in such a way that under the obvious identification of critical points with faces, the Morse theoretic differential agrees with the differential in the complex of singular cubes.

Now consider a smooth cube $\sigma:[-1,1]^{i} \rightarrow B$. Let $g$ be a fiberwise metric on the pullback bundle $\sigma^{*} Z \rightarrow[-1,1]^{i}$. Let $\xi$ denote the vector field on $\sigma^{*} Z$ given by the fiberwise negative gradient of $f$ with respect to $g$. 
Definition 3.2 Define a vector field $V$ on $\sigma^{*} Z$ by

$$
V:=\xi+H W_{i}
$$

Here $H$ denotes the horizontal lift via the pullback connection $\sigma^{*} \nabla$ on $\sigma^{*} Z$.

The critical points of $V$ are exactly the critical points of the functions $f_{b}$ on the fibers over the centers of the faces of $[-1,1]^{i}$.

Definition 3.3 A smooth cube $\sigma:[-1,1]^{i} \rightarrow B$ is admissible if $f_{b}$ is Morse whenever $b \in B$ is the center of a face of $\sigma$. The pair $(\sigma, g)$ is admissible if $\sigma$ is admissible and if the stable and unstable manifolds of the vector field $V$ on $\sigma^{*} Z$ intersect transversely.

Note that if $(\sigma, g)$ is admissible and $\sigma^{\prime}$ is a face of $\sigma$, then $\left(\sigma^{\prime},\left.g\right|_{\sigma^{\prime *} Z}\right)$ is also admissible. Furthermore, if $(\sigma, g)$ is admissible, then the orientations of the unstable manifolds of $W_{i}$, together with the orientations $\mathfrak{o}$, in that order, determine orientations of the unstable manifolds of $V$. If $p$ and $q$ are distinct critical points of $V$, let $\mathcal{M}(p, q)$ denote the moduli space of flow lines of $V$ from $p$ to $q$, ie maps $u: \mathbb{R} \rightarrow \sigma^{*} Z$ satisfying $u^{\prime}(s)=V(u(s)), \lim _{s \rightarrow-\infty} u(s)=p$, and $\lim _{s \rightarrow+\infty} u(s)=q$, modulo the $\mathbb{R}$ action by reparametrization. Admissibility implies that $\mathcal{M}(p, q)$ is a manifold. The orientations of the unstable manifolds of $V$ then determine an orientation of $\mathcal{M}(p, q)$, as in [35].

The following proposition, whose proof is deferred to Section 10, implies that if $\sigma$ is admissible, then $(\sigma, g)$ is admissible for generic $g$.

Proposition 3.4 (Genericity) Let $\sigma:[-1,1]^{i} \rightarrow B$ be an admissible cube, and let $g_{0}$ be a fiberwise metric on $(\partial \sigma)^{*} Z$. Assume that:

- each codimension one face $\sigma^{\prime}$ of $\sigma$, the pair $\left(\sigma^{\prime},\left.g_{0}\right|_{\sigma^{\prime *} Z}\right)$ is admissible.

Then $(\sigma, g)$ is admissible for a Baire set of fiberwise metrics $g$ on $\sigma^{*} Z$ extending $g_{0}$.

\subsection{The filtered chain complex}

We now define a bigraded chain complex $\left(\mathfrak{C}_{*, *}, \delta\right)$ as follows. If $f_{b}$ is Morse, let $\operatorname{Crit}_{j}(b):=\operatorname{Crit}_{j}\left(f_{b}\right)$ denote the set of index $j$ critical points of $f_{b}$.

Definition 3.5 (The bigraded complex) Let $\mathfrak{C}_{i, j}$ be the free $\mathbb{Z}$-module generated by triples $(\sigma, g, p)$, where:

- $\sigma:[-1,1]^{i} \rightarrow B$ is a smooth $i-$ cube, 
- $g$ is a metric on $\sigma^{*} Z$,

- $p \in \mathrm{Crit}_{j}(\sigma(0))$,

- the pair $(\sigma, g)$ is admissible.

When $i>0$, we mod out by triples $(\sigma, g, p)$ in which the pair $(\sigma, g)$ is degenerate, ie independent of at least one of the coordinates on $[-1,1]^{i}$.

Remark 3.6 One might instead try to define the fiberwise metric $g$ on $\sigma^{*} Z$ by pulling back a fixed, generic fiberwise metric $g_{Z}$ on $Z \rightarrow B$. However one would then want to show that the pair $\left(\sigma, \sigma^{*} g_{Z}\right)$ is admissible for generic $\sigma$, and this seems difficult.

Definition 3.7 (The differential) For $0 \leq k \leq i$, define

$$
\delta_{k}: \mathfrak{C}_{i, j} \longrightarrow \mathfrak{C}_{i-k, j+k-1}
$$

as follows. Let $(\sigma, g, p)$ be a generator of $\mathfrak{C}_{i, j}$. Let $F_{k}(\sigma)$ denote the set of codimension $k$ faces of $\sigma$. Then

$$
\delta_{k}(\sigma, g, p):=\sum_{\sigma^{\prime} \in F_{k}(\sigma), q \in \mathrm{Crit}_{j+k-1}\left(\sigma^{\prime}(0)\right)} \# \mathcal{M}(p, q) \cdot\left(\sigma^{\prime}, g_{\sigma^{\prime}}, q\right) .
$$

Here "\#" denotes the signed count using the orientation on $\mathcal{M}(p, q)$ specified previously; this is finite by the compactness argument in Proposition 3.9 below. Also, $g_{\sigma^{\prime}}$ denotes the restriction of $g$ to $\left(\sigma^{\prime}\right)^{*} Z$. Define the differential

$$
\delta:=\sum_{k=0}^{i} \delta_{k}: \mathfrak{C}_{i, j} \longrightarrow \bigoplus_{k=0}^{i} \mathfrak{C}_{i-k, j+k-1} .
$$

Example 3.8 $\delta_{0}$ is given by

$$
\delta_{0}(\sigma, g, p)=(-1)^{i}(\sigma, g, \partial p)
$$

where $\partial$ is the differential in the Morse complex $C_{*}^{\text {Morse }}(\sigma(0))$ for $\left(f_{\sigma(0)}, g_{0}\right)$. We have

$$
\delta_{1}(\sigma, g, p)=\sum_{\sigma^{\prime} \in F_{1}(\sigma)} \pm\left(\sigma^{\prime}, g_{\sigma^{\prime}}, \Phi(p)\right)
$$

where $\Phi: C_{*}^{\text {Morse }}(\sigma(0)) \rightarrow C_{*}^{\text {Morse }}\left(\sigma^{\prime}(0)\right)$ is the continuation homomorphism along the straight line in $[-1,1]^{i}$ from the center of $\sigma$ to the center of $\sigma^{\prime}$, defined using $\beta(t)=(1-t) t(1+t)$ in (3-1). The sign in (3-8) agrees with the sign of $\sigma^{\prime}$ in $\partial \sigma$. Also,

$$
\delta_{2}(\sigma, g, p)=\sum_{\sigma^{\prime} \in F_{2}(\sigma)}\left(\sigma^{\prime}, g_{\sigma^{\prime}}, K(p)\right)
$$


where $K: C_{*}^{\text {Morse }}(\sigma(0)) \rightarrow C_{*+1}^{\text {Morse }}\left(\sigma^{\prime}(0)\right)$ is a chain homotopy between the two compositions of continuation maps from $\sigma(0)$ to $\sigma^{\prime}(0)$ involving the centers of the two codimension 1 faces adjacent to $\sigma^{\prime}$.

The following proposition contains the facts that the Morse differential $\partial$ is a differential, the continuation maps $\Phi$ are chain maps, and $K$ is a chain homotopy, together with higher dimensional generalizations.

Proposition $3.9 \delta^{2}=0$.

Proof Let $\sigma^{\prime} \in F_{k}(\sigma)$ and $r \in \operatorname{Crit}_{j+k-2}\left(\sigma^{\prime}(0)\right)$. Similarly to (2-1), the moduli space $\mathcal{M}(p, r)$ has a compactification $\overline{\mathcal{M}(p, r)}$, which is a compact oriented one-manifold with boundary

$$
\partial \overline{\mathcal{M}(p, r)}=\bigcup_{\substack{\sigma^{\prime \prime} \in F_{k^{\prime}}(\sigma) \\ q \in \mathrm{Crit}_{j+k^{\prime}-1}\left(\sigma^{\prime \prime}(0)\right)}} \mathcal{M}(p, q) \times \mathcal{M}(q, r)
$$

as compact oriented 0 -manifolds.

For the compactness part of the proof of (3-9), to show that any sequence in $\mathcal{M}(p, r)$ has a subsequence converging to a broken flow line, we use the analogous fact for the moduli spaces of flow lines of $W_{i}$ in $[-1,1]^{i}$, together with an a priori upper bound on the "energy" of a flow line $u$ : $\mathbb{R} \rightarrow \sigma^{*} Z$, namely

$$
\int_{s=-\infty}^{s=+\infty}|\xi(u(s))|^{2}<f_{\sigma(0)}(p)-f_{\sigma^{\prime}(0)}(q)+C(\sigma)
$$

where $C(\sigma)$ is a constant. The upper bound (3-10) follows from

$$
\frac{d f}{d s}=-|\xi|^{2}+\nabla_{W_{i}} f_{\sigma}
$$

where $f_{\sigma}: \sigma^{*} Z \rightarrow \mathbb{R}$ denotes the function whose restriction to the fiber over $x \in[-1,1]^{i}$ is $f_{\sigma(x)}$.

Now the coefficient of $\left(\sigma^{\prime}, g_{\sigma^{\prime}}, r\right)$ in $\delta^{2}(\sigma, g, p)$ equals the number of points in the r.h.s. of Equation (3-9). Since the signed number of points in $\partial \overline{\mathcal{M}(p, r)}$ is zero for each $r$, it follows that $\delta^{2}(\sigma, g, p)=0$.

Definition 3.10 (Main definition) Define the chain complex

$$
\mathfrak{C}_{*}(Z):=\left(\bigoplus_{i+j=*} \mathfrak{C}_{i, j}, \delta\right)
$$

Algebraic $8 \mathcal{G}$ Geometric Topology, Volume 8 (2008) 
This has a filtration $F_{*} \mathfrak{C}_{*}$ defined by

$$
F_{i} \mathfrak{C}_{m}:=\bigoplus_{i^{\prime} \leq i} \mathfrak{C}_{i^{\prime}, m-i^{\prime}}
$$

Let $E_{*, *}^{*}(Z)$ denote the associated spectral sequence. Define the family Morse homology $H F_{*}(Z)$ to be the homology of $\mathfrak{C}_{*}$.

\section{Basic properties}

We now present a priori proofs that the spectral sequence $E_{*, *}^{*}$ defined in Section 3 for finite dimensional Morse theory satisfies the $E^{2}$, homotopy invariance, naturality, and triviality properties of the Main Principle (a)-(d). We further show that the family Morse homology $H F_{*}(Z)$ satisfies the Mayer-Vietoris property (g). These properties will also follow a posteriori from the comparison with the Leray-Serre spectral sequence in Theorem 5.1 (except for property (a) which is used in its proof). However, the $a$ priori proofs given here are quite simple and provide a model for demonstrating these properties of the spectral sequence for other versions of Floer theory, where a classical topological interpretation of the spectral sequence might not be available.

Proposition 4.1 ( $E^{2}$ term) There is a canonical identification

$$
E_{i, j}^{2}=H_{i}\left(B ; \mathcal{F}_{j}(Z)\right) .
$$

Proof Let $\widetilde{B}$ denote the space of pairs $(b, g)$, where $b \in B$ and $g$ is a metric on $Z_{b}$. Then $\widetilde{B}$ fibers over $B$ with contractible fibers. Moreover, a pair $(\sigma, g)$, where $\sigma$ is a smooth cube in $B$ and $g$ is a fiberwise metric on $\sigma^{*} Z$, is tautologically equivalent to a smooth cube $\widetilde{\sigma}:[-1,1]^{i} \rightarrow \widetilde{B}$.

Let $C_{*}\left(\widetilde{B} ; \mathcal{F}_{*}(Z)\right)$ denote the chain complex of smooth cubes in $\widetilde{B}$ with coefficients in the pullback of the local coefficient system $\mathcal{F}_{*}(Z)$ on $B$. Let $C_{*}^{\prime}\left(\widetilde{B} ; \mathcal{F}_{*}(Z)\right)$ denote the subcomplex defined using admissible pairs $(\sigma, g)$.

By equations (3-11) and (3-7), there is a canonical isomorphism of chain complexes

$$
E_{i, j}^{1}=C_{i}^{\prime}\left(\tilde{B} ; \mathcal{F}_{j}(Z)\right) .
$$

By Proposition 3.4, we can construct a chain homotopy by induction on the dimension to show that the inclusion $C_{i}^{\prime}\left(\widetilde{B} ; \mathcal{F}_{j}(Z)\right) \longrightarrow C_{i}\left(\widetilde{B} ; \mathcal{F}_{j}(Z)\right)$ induces an isomorphism on homology

$$
E_{i, j}^{2}=H_{i}\left(\widetilde{B} ; \mathcal{F}_{j}(Z)\right)
$$


Finally,

$$
H_{i}\left(\widetilde{B} ; \mathcal{F}_{j}(Z)\right)=H_{i}\left(B ; \mathcal{F}_{j}(Z)\right),
$$

since the fibers of $\widetilde{B} \rightarrow B$ are contractible.

Remark 4.2 (Comparing spectral sequences) Recall that a morphism of spectral sequences from $E_{*, *}^{*}$ to ${ }^{\prime} E_{*, *}^{*}$ defined from $E^{k}$ on is a map $\Phi_{r}: E_{i, j}^{r} \rightarrow{ }^{\prime} E_{i, j}^{r}$ defined for $r \geq k$ such that $\partial_{r}^{\prime} \Phi_{r}=\Phi_{r} \partial_{r}$ and $\Phi_{r+1}=\left(\Phi_{r}\right)_{*}$. A filtered chain map between filtered complexes induces a morphism of the associated spectral sequences from $E^{0}$ on. If a morphism of spectral sequences is an isomorphism on $E^{k}$, then by induction it is an isomorphism on the $E^{r}$ terms for all $r \geq k$. If a filtered chain map $\Phi$ induces an isomorphism on $E^{k}$, and if the spectral sequences converge, then $\Phi$ induces an isomorphism on homology.

If $Z$ is a family over $B$, then a smooth map $\phi: B^{\prime} \rightarrow B$ induces a pullback family $\phi^{*} Z$ over $B^{\prime}$. We continue to assume that $Z$ is admissible in the sense of Definition 3.1. It follows that a generic map $\phi$ is transverse to the strata in $B$ over which the fiberwise functions $f_{b}$ are not Morse, so that for generic $\phi$ the pullback family $\phi^{*} Z$ is also admissible.

Proposition 4.3 (Naturality) If $\phi: B^{\prime} \rightarrow B$ is generic so that $\phi^{*} Z$ is admissible, then the pushforward in homology

$$
\phi_{*}: H_{*}\left(B^{\prime} ; \mathcal{F}_{*}\left(\phi^{*} Z\right)\right) \longrightarrow H_{*}\left(B ; \mathcal{F}_{*}(Z)\right)
$$

extends to a morphism of spectral sequences from $E^{2}$ on,

$$
\phi_{*}: E_{*, *}^{*}\left(\phi^{*} Z\right) \longrightarrow E_{*, *}^{*}(Z) .
$$

Proof If $\sigma:[-1,1]^{i} \rightarrow B^{\prime}$ is a cube, then there is a tautological identification of pullback bundles over $[-1,1]^{i}$ :

$$
(\phi \circ \sigma)^{*} Z=\sigma^{*}\left(\phi^{*} Z\right) \text {. }
$$

Moreover, $\sigma$ is admissible for $\phi^{*} Z$ if and only if $\phi \circ \sigma$ is admissible for $Z$. Hence we have a well-defined filtered chain map,

$$
\begin{aligned}
\phi_{*}: \mathfrak{C}_{i, j}\left(\phi^{*} Z\right) & \longrightarrow \mathfrak{C}_{i, j}(Z), \\
(\sigma, g, p) & \longmapsto(\phi \circ \sigma, g, p) .
\end{aligned}
$$

By Remark 4.2, this induces a morphism of spectral sequences from $E^{0}$ on. By the identifications in the proof of Proposition 4.1, the map on the $E^{2}$ terms is given by (4-3). 
As explained in Section 1.2, the previous two propositions imply that the spectral sequence $E_{*, *}^{*}(Z)$ is homotopy invariant from the second term on, and the induced maps on it are functorial and homotopy invariant. The argument can also be modified to establish the same conclusions for the family Morse homology $H F_{*}(Z)$ and the induced maps on it. We omit the details of this, because stronger statements are proved in Section 8. For now let us prove:

Proposition 4.4 (Mayer-Vietoris) $H F_{*}(Z)=H_{*}\left(\mathfrak{C}_{*}\right)$ satisfies property $(g)$ in the Main Principle.

Proof Let $\mathfrak{C}_{*}^{\prime}$ denote the subcomplex of $\mathfrak{C}_{*}(Z)$ defined using only cubes $\sigma$ whose image is contained in $U$ or $V$. By the local coefficient version of the standard subdivision lemma (cf [19]), the proof of Proposition 4.1 shows that the inclusion $\mathfrak{C}_{*}^{\prime} \rightarrow \mathfrak{C}_{*}$ induces an isomorphism on the $E^{2}$ terms of the associated spectral sequences, and hence an isomorphism on homology. The short exact sequence of chain complexes

$$
0 \longrightarrow \mathfrak{C}_{*}\left(\left.Z\right|_{U \cap V}\right) \longrightarrow \mathfrak{C}_{*}\left(\left.Z\right|_{U}\right) \oplus \mathfrak{C}_{*}\left(\left.Z\right|_{V}\right) \longrightarrow \mathfrak{C}_{*}^{\prime} \longrightarrow 0
$$

now induces the desired long exact sequence in homology.

Proposition 4.5 (Triviality) If $Z=B \times X$ and $f_{b}: X \rightarrow \mathbb{R}$ is the same Morse function $f_{X}$ for all $b \in B$, then the spectral sequence $E_{*, *}^{*}(Z)$ collapses at $E^{2}$.

Proof By homotopy invariance, we can choose $\nabla$ to be the trivial connection, and we can choose $\mathfrak{o}$ to be the same orientation of the descending manifolds over each fiber. Fix a metric $g_{X}$ on $X$ such that the pair $\left(f_{X}, g_{X}\right)$ is Morse-Smale. Consider the subcomplex $\widehat{\mathfrak{C}}_{*}$ of the filtered complex $\mathfrak{C}_{*}$ spanned by triples $\left(\sigma, \sigma^{*} g_{X}, p\right)$. Note that the pair $\left(\sigma, \sigma^{*} g_{X}\right)$ is admissible, because any flow line of the vector field $V$ on $\sigma^{*} Z=[-1,1]^{i} \times X$ projects to a flow line in $X$ of the negative gradient of $f_{X}$ with respect to $g_{X}$. Moreover the moduli spaces that contribute to $\delta_{k}\left(\sigma, \sigma^{*} g_{X}, p\right)$ for $k \geq 2$ are empty on dimensional grounds. Hence, by equations (3-7) and (3-8), the subcomplex $\widehat{\mathfrak{C}}_{*}$ is the tensor product of the smooth cubical singular complex of $B$ and the Morse complex for $\left(f_{X}, g_{X}, \mathfrak{o}\right)$. It follows as in the algebraic Künneth formula that the spectral sequence for $\widehat{\mathfrak{C}}_{*}$ collapses at $E^{2}$. To finish the proof, the inclusion of filtered complexes $\widehat{\mathfrak{C}}_{*} \rightarrow \mathfrak{C}_{*}$ induces a morphism of spectral sequences which is an isomorphism on $E^{2}$, and hence from $E^{2}$ on.

There is also the following variant of Proposition 4.5. 
Proposition 4.6 (Obstruction to simultaneous regularity) Let $Z$ be a family such that $f_{b}: Z_{b} \rightarrow \mathbb{R}$ is Morse for every $b \in B$. Suppose there exists a fiberwise metric $g$ on $Z$ such that $\left(f_{b}, g_{b}\right)$ is Morse-Smale for each $b \in B$. Then $E_{*, *}^{*}(Z)$ collapses at $E^{2}$ over $\mathbb{Q}$, and over $\mathbb{Z}$ if $B$ is simply connected.

Proof Over any cube $\sigma:[-1,1]^{i} \rightarrow B$, we can identify the Morse complexes for all fibers of $\sigma^{*} Z$. We claim that if $\left(\sigma, g^{\prime}\right)$ is admissible, if $\sigma$ is sufficiently $C^{1}$-close to a constant cube, and if $g^{\prime}$ is sufficiently close to $\sigma^{*} g$, then for $\alpha$ in the fiberwise Morse complex,

$$
\delta\left(\sigma, g^{\prime}, \alpha\right)=(-1)^{i}\left(\sigma, g^{\prime}, \partial \alpha\right)+\sum_{\sigma^{\prime} \in F_{1}(\sigma)} \pm\left(\sigma^{\prime}, g_{\sigma^{\prime}}^{\prime}, \alpha\right) .
$$

Here " \pm " agrees with the sign of $\sigma^{\prime}$ in $\partial \sigma$.

To prove the claim, first note that if $\sigma$ is a constant cube $\sigma_{b}:[-1,1]^{i} \rightarrow\{b\}$ and $g^{\prime}=\sigma_{b}^{*} g$, then $\left(\sigma, g^{\prime}\right)=\left(\sigma_{b}, \sigma_{b}^{*} g\right)$ is admissible and satisfies (4-5), as in the proof of Proposition 4.5. (Here we are temporarily forgetting to mod out by degenerate cubes.) Now all the flow lines that contribute to $\delta\left(\sigma_{b}, \sigma_{b}^{*} g, \alpha\right)$ are transverse and so persist under a $C^{1}$-small perturbation of $\left(\sigma_{b}, \sigma_{b}^{*} g\right)$ to $\left(\sigma, g^{\prime}\right)$. On the other hand, no other flow lines can contribute to (4-5) when the perturbation is sufficiently small. Otherwise we can take a limit in which the perturbation shrinks to zero and obtain an index one broken flow line $\widehat{u}$ for $\left(\sigma_{b}, \sigma_{b}^{*} g\right)$. Then $\widehat{u}$ must be unbroken, or else it would contain a component living in a moduli space of negative dimension. Hence $\widehat{u}$ agrees with one of the flow lines that we have already accounted for in (4-5). This proves the claim.

By subdividing cubes and using Propositions 3.4 and 4.1, one can represent any element of $E^{2}$ by a sum of generators $\left(\sigma, g^{\prime}, \alpha\right)$ for which (4-5) holds. The conclusion of the proposition now follows as in [4, Section 5.6], where the corresponding statement is proved for the Leray-Serre spectral sequence.

\section{Comparison with Leray-Serre}

We now show that the spectral sequence constructed above agrees with the Leray-Serre spectral sequence.

Given a family $Z$ as before, let $C_{*}(Z)$ denote the cubical singular chain complex of the total space $Z$. Let $F_{i} C_{i+j}(Z)$ denote the subcomplex generated by singular $(i+j)$-cubes $\sigma:[-1,1]^{i+j} \rightarrow Z$ such that the composition $\pi \circ \sigma:[-1,1]^{i+j} \rightarrow B$ is independent of at least $j$ of the coordinates on $[-1,1]^{i+j}$. The filtered complex $F_{*} C_{*}(Z)$ gives rise to the (homological) Leray-Serre spectral sequence, which we 
denote here by $L S_{*, *}^{*}(Z)$ and regard as defined from $L S^{2}$ on. It satisfies $L S_{i, j}^{2}(Z) \simeq$ $H_{i}\left(B ;\left\{H_{j}\left(Z_{b}\right)\right\}\right)$ and converges to the homology of $Z$. We now have the following generalization of the fundamental isomorphism (2-5).

Theorem 5.1 Let $\pi: Z \rightarrow B$ be a smooth fiber bundle whose fibers are closed manifolds. Then:

(a) The Morse theory spectral sequence $E_{i, j}^{k}(Z)$ and the Leray-Serre spectral sequence $L S_{i, j}^{k}(Z)$ are canonically isomorphic for $k \geq 2$.

(b) The family Morse homology $H F_{*}(Z)$ is isomorphic to the singular homology of the total space, $H_{*}(Z)$.

Proof We define a filtered chain map

$$
\Psi: \mathfrak{C}_{i, j} \rightarrow F_{i} C_{i+j}(Z)
$$

as follows. Let $(\sigma, g, p)$ be a generator of $\mathfrak{C}_{i, j}$. Let $\mathcal{D}(p) \subset \sigma^{*} Z$ denote the unstable manifold of $p$ with respect to the vector field $V$ defined in Equation (3-5). Let $e: \overline{\mathcal{D}}(p) \rightarrow \sigma^{*} Z$ be its compactification as in Section 2.3. As in Equation (2-7), we have

$$
\partial \overline{\mathcal{D}(p)}=\bigcup_{q \in \operatorname{Crit}(V)}(-1)^{\operatorname{ind}_{V}(p)-\operatorname{ind}_{V}(q)-1} \mathcal{M}(p, q) \times \overline{\mathcal{D}(q)}
$$

Let $\overline{[-1,1]^{i}}$ denote the analogous compactification of the unstable manifold of 0 with respect to the flow $W_{i}$. For example, $\overline{[-1,1]^{3}}$ is diffeomorphic to a cube which is "fully truncated" by replacing the vertices, edges, and faces with hexagons, rectangles, and octagons respectively. Since flow lines of $V$ project to flow lines of $W_{i}$, the projection $\sigma^{*} Z \rightarrow[-1,1]^{i}$ induces a continuous map $\bar{\pi}$ making the diagram

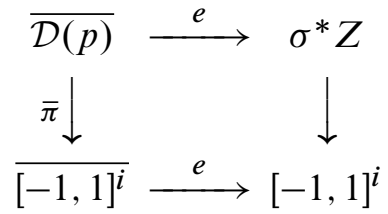

commute. One can check, using the local parametrizations of the manifolds with corners as in [2], that the map $\bar{\pi}$ is a Serre fibration.

It follows that the fundamental class of $\overline{\mathcal{D}(p)}$ can be represented by a cubical singular chain $d_{p}$ consisting of cubes $\sigma^{\prime}$ such that $\bar{\pi} \circ \sigma^{\prime}$ is independent of $j$ of the coordinates on $[-1,1]^{i+j}$. As in Section 2.3, by Equation (5-2) we can choose these chains by 
induction on the dimension so that the analogue of Equation (2-9) holds. Letting $\imath: \sigma^{*} Z \rightarrow Z$ denote the natural map, we finally define

$$
\Psi(\sigma, g, p):=(l \circ e)_{*}\left(d_{p}\right) .
$$

Then $\Psi$ respects the filtrations and $\partial \Psi=\Psi \delta$. Hence $\Psi$ induces a morphism from the Morse theory spectral sequence to the Leray-Serre spectral sequence defined from $E^{0}$ on.

(a) We claim that this morphism of spectral sequences is a canonical isomorphism from $E^{2}$ on. By Remark 4.2, it will suffice to show that the map on $E^{2}$ terms

$$
\Psi_{*}: H_{i}\left(B ; \mathcal{F}_{j}(Z)\right) \stackrel{\simeq}{\longrightarrow} H_{i}\left(B ;\left\{H_{j}\left(Z_{b}\right)\right\}\right)
$$

is a canonical isomorphism. In fact, the map (5-3) is the isomorphism induced by the canonical isomorphism of local coefficient systems (3-2). The reason is that if $\alpha$ is an element of the Morse homology over the center of $\sigma$, then by construction, the intersection of $e_{*}\left(d_{p}\right)$ with any fiber over the interior of $\sigma$ or the interior of a codimension one face agrees with the image of $\alpha$ under the canonical map (2-5) from Morse homology to singular homology.

(b) Since the filtered chain map (5-1) induces an isomorphism of spectral sequences, it induces an isomorphism on homology.

One can also show that the isomorphism in (b) on homology is canonical. That is, it does not depend on the choice of filtered chain map (5-1), and it commutes with the isomorphisms on family Morse homology given by the homotopy invariance in Proposition 8.6 below. The details of this are omitted.

\section{Alternate construction}

We now give alternate constructions of the spectral sequence and family Floer homology for a family $(\pi: Z \rightarrow B, f, \nabla)$ as in Section 3.1, in the special case when the base $B$ is a closed manifold. The alternate constructions use Morse homology on $B$ instead of singular homology, and are considerably simpler. We then show that the alternate constructions for this special case agree with the original ones.

\subsection{A simpler filtered chain complex}

Fix a Morse function $f^{B}: B \rightarrow \mathbb{R}$ such that the fiberwise function $f_{x}$ is Morse for each $x \in \operatorname{Crit}\left(f^{B}\right)$. Fix a metric $g^{B}$ on $B$ such that the pair $\left(f^{B}, g^{B}\right)$ is Morse-Smale. 
Let $\mathcal{W}$ denote the negative gradient of $f^{B}$ with respect to $g^{B}$, and choose orientations $\mathfrak{o}^{B}$ of the descending manifolds of the critical points of $f^{B}$. Let $g^{Z}$ be a fiberwise metric on $Z$ and let $\xi$ denote the fiberwise negative gradient of $f$ with respect to $g^{Z}$. Define a vector field $\mathcal{V}$ on $Z$ by

$$
\mathcal{V}:=\xi+H \mathcal{W}
$$

where $H$ denotes horizontal lift with respect to $\nabla$. The critical points of $\mathcal{V}$ can be identified with pairs $(x, p)$ where $x \in B$ is a critical point of $f^{B}$ and $p$ is a critical point of $f_{x}$.

For generic fiberwise metrics $g^{Z}$, the stable and unstable manifolds of $\mathcal{V}$ intersect transversely. The proof of this is the same as the proof of Proposition 3.4 given in Section 10, except that we replace the Morse-Smale vector field $W_{i}$ on $[-1,1]^{i}$ with the Morse-Smale vector field $\mathcal{W}$ on $B$, and we do not fix the fiberwise metric over a subset of the base.

Assume now that $g^{Z}$ is generic in this sense. If $p$ and $q$ are critical points of $\mathcal{V}$, let $\mathcal{M}(p, q)$ denote the moduli space of flow lines of $\mathcal{V}$ from $p$ to $q$, modulo reparametrization. The chosen orientations $\mathfrak{o}^{B}$ and $\mathfrak{o}$, in that order, determine orientations of the unstable manifolds of $\mathcal{V}$, which in turn determine orientations of the moduli spaces $\mathcal{M}(p, q)$.

Now define a bigraded chain complex $\left(\mathcal{C}_{i, j}, \delta\right)$ as follows. The chain module $\mathcal{C}_{i, j}$ is the free $\mathbb{Z}$-module generated by pairs $(x, p)$, where $x \in \operatorname{Crit}_{i}\left(f^{B}\right)$ and $p \in \operatorname{Crit}_{j}\left(f_{x}\right)$. For $k \geq 0$ define $\delta_{k}: \mathcal{C}_{i, j} \rightarrow \mathcal{C}_{i-k, j+k-1}$ by

$$
\delta_{k}(x, p):=\sum_{y \in \mathrm{Crit}_{i-k}\left(f^{B}\right), q \in \operatorname{Crit}_{j+k-1}\left(f_{y}\right)} \# \mathcal{M}((x, p),(y, q)) \cdot(y, q) .
$$

We then define $\delta:=\sum_{k \geq 0} \delta_{k}$, and the usual arguments show that $\delta$ is well-defined and $\delta^{2}=0$. Thus as in Section 3.3 we have a filtered chain complex, which we denote by $\mathcal{C}_{*}\left(Z, g^{Z}, f^{B}, g^{B}\right)$.

Denote the associated spectral sequence $\mathcal{E}_{*, *}^{*}\left(Z, g^{Z}, f^{B}, g^{B}\right)$. The first term of the spectral sequence is given by the Morse complex of $\left(f^{B}, g^{B}\right)$ with local coefficients in the sheaf of Morse homologies $\mathcal{F}_{*}(Z)$ :

$$
\mathcal{E}_{i, j}^{1}=C_{i}^{\text {Morse }}\left(f^{B}, g^{B} ; \mathcal{F}_{j}(Z)\right) .
$$

Hence, by the local coefficient version of the fundamental isomorphism (2-5),

$$
\mathcal{E}_{i, j}^{2}=H_{i}\left(B ; \mathcal{F}_{j}(Z)\right) .
$$




\subsection{Equivalence of the two spectral sequences}

We now have two spectral sequences $E_{*, *}^{*}$ and $\mathcal{E}_{*, *}^{*}$ when $B$ is a closed manifold, defined using cubical singular homology and Morse homology on $B$ respectively. The following proposition shows that they are isomorphic. To start, by (4-1) and (6-2), we have a canonical identification

$$
E_{i, j}^{2}=\mathcal{E}_{i, j}^{2}
$$

Proposition 6.1 When the base $B$ is a closed manifold, the identification (6-3) extends to a canonical isomorphism of spectral sequences for $k \geq 2$,

$$
E_{i, j}^{k}(Z)=\mathcal{E}_{i, j}^{k}\left(Z, g^{Z}, f^{B}, g^{B}\right) .
$$

Proof Let $\mathfrak{C}_{*}^{\prime}$ denote the subcomplex of $\mathfrak{C}_{*}$ spanned by triples $(\sigma, g, p)$ such that the cube $\sigma$, and all of its faces, are transverse to the ascending manifolds of $\left(f^{B}, g^{B}\right)$. Since any cubical chain in $B$ can be perturbed so that all cubes satisfy the above transversality assumption, the inclusion $\mathfrak{C}_{*}^{\prime \prime} \rightarrow \mathfrak{C}_{*}^{*}$ induces an isomorphism of spectral sequences from the second term on by the argument of Proposition 4.1. (In fact Lemma 8.5 below implies that the inclusion $\mathfrak{C}_{*}^{\prime} \rightarrow \mathfrak{C}_{*}$ is a filtered chain homotopy equivalence.) To prove the proposition, we will define a filtered chain map

$$
\bar{\Xi}: \mathfrak{C}_{*}^{\prime \prime} \longrightarrow \mathcal{C}_{*}\left(Z, g^{Z}, f^{B}, g^{B}\right)
$$

which induces the map (6-3) on the $E^{2}$ term. This will be a variant of the map (2-10) from singular homology to Morse homology on $B$.

Let $\phi_{t}: B \rightarrow B$ denote the time $t$ flow of the negative gradient $\mathcal{W}$ of $\left(f^{B}, g^{B}\right)$. For any cube $\sigma:[-1,1]^{i} \rightarrow B$, its forward orbit is the map

$$
\begin{aligned}
\bar{\sigma}:(0, \infty) \times[-1,1]^{i} & \longrightarrow B, \\
(t, x) & \longmapsto \phi_{t}(\sigma(x)) .
\end{aligned}
$$

Consider the pullback bundle

$$
\bar{\sigma}^{*} Z \longrightarrow(0, \infty) \times[-1,1]^{i} .
$$

There is a tautological map $e: \bar{\sigma}^{*} Z \rightarrow Z$. For each admissible pair $(\sigma, g)$ where $\sigma$ satisfies the above transversality conditions, choose a fiberwise metric $\bar{g}$ for the pullback bundle $\bar{\sigma}^{*} Z$ which limits to $g$ as the $(0, \infty)$ coordinate $t$ goes to zero, and which agrees with $e^{*} g^{Z}$ for $t \geq 1$. Let $\bar{\xi}$ denote the fiberwise negative gradient of the fiberwise function $f$ with respect to $\bar{g}$ on $\bar{\sigma}^{*} Z$. 
Choose a monotone smooth function $\rho:(0, \infty) \rightarrow \mathbb{R}$ with $\rho(t)=t$ for $t<1 / 2$ and $\rho(t)=1$ for $t \geq 1$. For $(\sigma, g)$ as above, consider the vector field

$$
\bar{V}:=\bar{\xi}+H\left(\rho(t) \frac{\partial}{\partial t}+(1-\rho(t)) W_{i}\right)
$$

on the pullback bundle $\bar{\sigma}^{*} Z$. Here $H$ denotes the horizontal lift with respect to the connection $\nabla$ and $t$ denotes the $(0, \infty)$ coordinate. Note that the vector field (6-5) interpolates between (3-5) and (6-1), in that $\bar{V}$ agrees with $V$ in the limit as $t \rightarrow 0$, while $e_{*} \bar{V}=\mathcal{V}$ when $t \geq 1$.

If $(\sigma, g, p)$ is a generator of $\mathfrak{C}_{i, j}^{\prime}$ and $(x, q)$ is a generator of $\mathcal{C}_{i^{\prime}, j^{\prime}}$, denote by $\mathcal{M}((\sigma, g, p),(x, q))$ the moduli space of flow lines $u: \mathbb{R} \rightarrow \bar{\sigma}^{*} Z$ of $\bar{V}$ such that $\lim _{s \rightarrow-\infty} u(s)=((0,0), p)$ and $\lim _{s \rightarrow \infty} e(u(s))=(x, q)$, modulo precomposition with translations of $\mathbb{R}$. Similarly to Proposition 3.4 , we can choose the fiberwise metrics $\bar{g}$ by induction on $i$ so that they are compatible with the face maps and so that these flow lines of the vector field $\bar{V}$ are cut out transversely. Then

$$
\operatorname{dim} \mathcal{M}((\sigma, g, p),(x, q))=(i+j)-\left(i^{\prime}+j^{\prime}\right) .
$$

If $u$ is such a flow line of $V$, then the projection $t \circ u: \mathbb{R} \rightarrow(0, \infty)$ is bijective. Thus $\bar{\sigma}$ sends the portion of $u$ with $t \in[1, \infty)$ to a flow line of $\mathcal{W}$ in $B$ from $\left(\phi_{1}\right)_{*} \sigma$ to $x$. So in the notation of Section 2.3, there is a map

$$
\mathcal{M}((\sigma, g, p),(x, q)) \longrightarrow \mathcal{M}\left(\left(\phi_{1}\right)_{*} \sigma, x\right)
$$

In particular, the orientation of $\mathcal{M}\left(\left(\phi_{1}\right)_{*} \sigma, x\right)$, together with the orientations $\mathfrak{o}$ and $\mathfrak{o}^{B}$ of the descending manifolds of $p$ and $q$, determine an orientation of $\mathcal{M}((\sigma, g, p),(x, q))$.

If $i+j=k$ and $i^{\prime}+j^{\prime}=k-1$, then the usual arguments show that the moduli space $\mathcal{M}((\sigma, g, p),(x, q))$ has a compactification to a 1 -manifold with boundary

$$
\begin{gathered}
\partial \overline{\mathcal{M}}((\sigma, g, p),(x, q)) \\
=\underset{y \in \operatorname{Crit}_{i^{\prime \prime}}\left(f^{B}\right), r \in \mathrm{Crit}_{k-i^{\prime \prime}}\left(f_{y}\right)}{\bigcup} \bigcup_{\sigma^{\prime} \in F_{i-i^{\prime \prime}}(\sigma),} \bigcup_{r \in \mathrm{Crit}_{k-i^{\prime \prime}-1}\left(f_{\sigma^{\prime}(0)}\right)} \mathcal{M}(p, r) \times \mathcal{M}\left(\left(\sigma^{\prime}, g_{\sigma^{\prime}}, r\right),(x, q)\right) .
\end{gathered}
$$

The point is that if $\left\{u_{n}\right\}$ is a sequence of flow lines in $\mathcal{M}((\sigma, g, p),(x, q)$ with no convergent subsequence, then the projection of each $u_{n}$ to $(0, \infty) \times[-1,1]^{i}$ hits $\left(1, z_{n}\right)$ for a unique $z_{n} \in[-1,1]^{i}$. We can pass to a subsequence such that $z_{n}$ converges to $z_{\infty} \in[-1,1]^{i}$. If $z_{\infty} \in \operatorname{int}[-1,1]^{i}$ or $z_{\infty} \in \partial[-1,1]^{i}$, then $u_{n}$ converges in appropriate 
sense to an element of the first term on the right side of (6-7) or the second term, respectively.

Now define $\bar{\Xi}: \mathfrak{C}_{*}^{\prime \prime} \rightarrow \mathcal{C}_{*}$ by

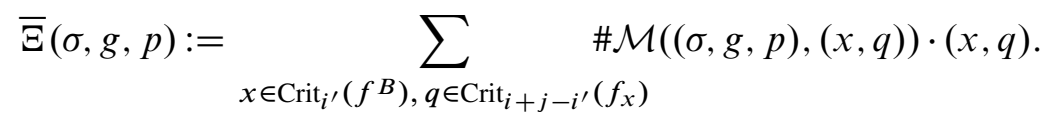

It follows from (6-7) that $\bar{\Xi}$ is a chain map. Moreover, $\bar{\Xi}$ respects the filtrations, because if $\mathcal{M}((\sigma, g, p),(x, q)) \neq \varnothing$, then by $(6-6), \mathcal{M}\left(\phi_{1 *} \sigma, x\right) \neq \varnothing$, so $\mathcal{M}(\sigma, x) \neq$ $\varnothing$, whence by our transversality assumptions $i^{\prime} \leq i$. On the $E^{2}$ term, $\bar{\Xi}$ induces the composition

$$
E_{i, j}^{2}=H_{*}\left(B ; \mathcal{F}_{j}(Z)\right) \stackrel{\phi_{1 *}}{\longrightarrow} H_{*}\left(B ; \mathcal{F}_{j}(Z)\right) \stackrel{\Xi_{*}}{\longrightarrow} H_{i}^{\text {Morse }}\left(f^{B}, g^{B} ; \mathcal{F}_{j}(Z)\right)=\mathcal{E}_{i, j}^{2} .
$$

Here $\Xi$ denotes the local coefficient version of the map (2-10) from singular homology to Morse homology. Since $\phi_{1 *}$ is the identity, the above composition is the canonical identification (6-3).

Remark 6.2 When $B=S^{k}$ with $k>1$, to show that the spectral sequence is equivalent to the construction described in Remark 1.10, one can use a Morse function $f^{B}: S^{k} \rightarrow \mathbb{R}$ with two critical points and take a limit in which the two critical points converge to each other.

Remark 6.3 The spectral sequence $\mathcal{E}_{*, *}^{*}$ is similar to the Morse-theoretic construction of the Leray-Serre spectral sequence in [31]. Another related way to obtain the LeraySerre spectral sequence Morse-theoretically is as the spectral sequence associated to the Morse-Bott function $\pi^{*} f^{B}$ on $Z$; $\operatorname{cf}[1 ; 5 ; 15 ; 27 ; 31]$. Our construction differs in its emphasis on starting with a family of smooth functions on the fibers, in order to enable generalizations to Floer homology of families.

\subsection{Alternate definition of family Morse homology}

Recall that the family Morse homology $H F_{*}(Z)$ is defined to be the homology of the complex $\mathfrak{C}_{*}(Z)$. When the base $B$ is a closed manifold, one could alternatively define the family Morse homology to be the homology of the complex $\mathcal{C}_{*}\left(Z, g^{Z}, f^{B}, g^{B}\right)$. Denote the latter homology by $H F_{*}^{\prime}(Z)$. We now show that this does not depend on $g^{Z}, f^{B}$, or $g^{B}$. Moreover, the filtered chain homotopy type of the complex $\mathcal{C}_{*}$ is also independent of these choices, and homotopy invariant: 
Proposition 6.4 Let $(\pi: Z \rightarrow[0,1] \times B, f, \nabla)$ be a family as in Section 3.1, where $B$ is a closed manifold. Assume that $Z_{0}:=\left.Z\right|_{\{0\} \times B}$ and $Z_{1}:=\left.Z\right|_{\{1\} \times B}$ are admissible. For $t=0,1$, let $\left(f_{t}^{B}, g_{t}^{B}\right)$ be Morse-Smale pairs on $B$ as in Section 6.1, and let $g_{t}^{Z}$ be a generic fiberwise metric on $Z_{t}$. Then there is a canonical (up to filtered chain homotopy) filtered chain homotopy equivalence

$$
\Phi: \mathcal{C}_{*}\left(Z_{0}, g_{0}^{Z}, f_{0}^{B}, g_{0}^{B}\right) \longrightarrow \mathcal{C}_{*}\left(Z_{1}, g_{1}^{Z}, f_{1}^{B}, g_{1}^{B}\right) .
$$

Proof The proof is a version of the standard continuation argument. There are three steps.

Step 1 Define a filtered chain map (6-8) as follows. First extend $\left(f_{0}^{B}, g_{0}^{B}\right)$ and $\left(f_{1}^{B}, g_{1}^{B}\right)$ to a generic smooth family of pairs of functions and metrics $\left\{\left(f_{t}^{B}, g_{t}^{B}\right)\right\}_{t \in[0,1]}$ on $B$. Let $\mathcal{W}_{t}$ denote the negative $g_{t}^{B}$-gradient of $f_{t}^{B}$. Next, extend $g_{0}^{Z}$ and $g_{1}^{Z}$ to a generic fiberwise metric $g^{Z}$ on $Z$ over $[0,1] \times B$. Let $\xi_{t}$ denote the fiberwise negative gradient of $f$ with respect to $g^{Z}$ over $\{t\} \times B$. Now define a vector field $\overline{\mathcal{V}}$ on $Z$ as follows: If $t \in[0,1], x \in B$, and $y \in Z_{(t, x)}$, then

$$
\overline{\mathcal{V}}(t, x, y):=\beta(t) \partial_{t}+\xi_{t}+H_{t} \mathcal{W}_{t} .
$$

Here $\beta$ is the function chosen in Section 2.2, and $H_{t}$ denotes the horizontal lift with respect to the restriction of $\nabla$ to $\{t\} \times B$. The map $\Phi$ is then defined by counting flow lines of $\overline{\mathcal{V}}$ with signs. (Our convention for defining these signs is to orient the descending manifolds of $\overline{\mathcal{V}}$ using the $t$ coordinate first, then the orientations of the descending manifolds of the Morse functions $f_{t}^{B}$ on the base, and finally the orientations of the descending manifolds of the fiberwise Morse functions.) As in Proposition 3.4, if $g^{Z}$ is generic then $\Phi$ is well defined. The usual consideration of ends of 1-dimensional moduli spaces of flow lines of $\overline{\mathcal{V}}$ shows that $\Phi$ is a chain map. Moreover, $\Phi$ preserves the filtration, because any flow line of $\overline{\mathcal{V}}$ projects to a flow line of the vector field $(2-2)$ on $[0,1] \times B$ that defines the continuation map $C_{*}^{\text {Morse }}\left(f_{0}^{B}, g_{0}^{B}\right) \rightarrow C_{*}^{\text {Morse }}\left(f_{1}^{B}, g_{1}^{B}\right)$.

Step 2 We now show that up to filtered chain homotopy, the "continuation map" (6-8) is independent of choices and invariant under homotopy of the family $Z$.

Consider a family $\pi: \bar{Z} \rightarrow[0,1]^{2} \times B$, such that $\left.\bar{Z}\right|_{[0,1] \times\{0\} \times B}$ and $\left.\bar{Z}\right|_{[0,1] \times\{1\} \times B}$ are pulled back from $Z_{0}$ and $Z_{1}$ respectively. For $s=0,1$, let $\left\{\left(f_{s, t}^{B}, g_{s, t}^{B}\right)\right\}_{t \in[0,1]}$ be a generic family of functions and metrics on $B$, and let $\bar{g}_{s}$ be a generic fiberwise metric on $\bar{Z}_{\{s\} \times[0,1] \times B}$, such that for $t=0,1$ we have $\left(f_{s, t}^{B}, g_{s, t}^{B}\right)=\left(f_{t}^{B}, g_{t}^{B}\right)$ and $\left.\bar{g}_{s}\right|_{\{s\} \times\{t\} \times B}=g_{t}^{Z}$. For $s=0,1$, our chosen data over $\{s\} \times[0,1] \times B$ then define a continuation map $\Phi_{s}$ as in (6-8). We claim that $\Phi_{0}$ and $\Phi_{1}$ are filtered chain homotopic. 
To see this, choose a family of pairs $\left\{\left(f_{s, t}^{B}, g_{s, t}^{B}\right)\right\}_{(s, t) \in[0,1]^{2}}$, and a generic fiberwise metric $g^{\bar{Z}}$ on $\bar{Z}$, extending our choices on the boundary of the square. Define a module homomorphism

$$
K: \mathcal{C}_{*}\left(Z_{0}, g_{0}^{Z}, f_{0}^{B}, g_{0}^{B}\right) \longrightarrow \mathcal{C}_{*+1}\left(Z_{1}, g_{1}^{Z}, f_{1}^{B}, g_{1}^{B}\right)
$$

by counting flow lines of the vector field

$$
\beta(t) \partial_{t}+\xi_{s, t}+H_{s, t} \mathcal{W}_{s, t}
$$

on $\bar{Z}$ with signs. By the usual arguments, if $g^{\bar{Z}}$ is generic then $K$ is well-defined, and for appropriate sign conventions in the counting satisfies

$$
\delta K+K \delta=\Phi_{0}-\Phi_{1} .
$$

Moreover, $K$ increases the filtration by at most 1 , because any flow line of the vector field (6-10) projects to a flow line of the vector field (2-4) on $[0,1]^{2} \times B$ that defines the chain homotopy between continuation maps on the Morse homology of $B$.

Step 3 Similarly to Step 2, the continuation map induced by a family $\widehat{Z}$ over [0, 2] $\times B$ is filtered chain homotopic to the composition of the continuation maps induced by the restrictions of $\widehat{Z}$ to $[0,1] \times B$ and $[1,2] \times B$. Also, if the family $Z$ over $[0,1] \times B$ is pulled back from a family over $B$, if $g_{0}^{Z}=g_{1}^{Z}$, and if $\left(f_{0}^{B}, g_{0}^{B}\right)=\left(f_{1}^{B}, g_{1}^{B}\right)$, then in Step 1 one can take $\left(f_{t}^{B}, g_{t}^{B}\right)$ and the fiberwise metric $g^{Z}$ to be independent of $t$, and one obtains $\Phi=\mathbb{1}$. These formal properties of $\Phi$ imply the proposition.

\subsection{Equivalence of the two family Morse homologies}

Proposition 6.5 If the base $B$ of the family $Z$ is a closed manifold, then there is a canonical isomorphism of filtered modules

$$
H F_{*}(Z)=H F_{*}^{\prime}(Z) .
$$

Proof To define an isomorphism, fix data $\left(g^{Z}, f^{B}, g^{B}\right)$ to define $\mathcal{C}_{*}$. As in Section 6.2 , let $\mathfrak{C}_{*}^{\prime \prime}$ denote the subcomplex of $\mathfrak{C}_{*}$ defined using admissible cubes $\sigma$ such that all faces of $\sigma$ are transverse to the ascending manifolds of $f^{B}$ with respect to $g^{B}$. Recall that the inclusion $\mathfrak{C}_{*}^{\prime} \rightarrow \mathfrak{C}_{*}$ induces an isomorphism on homology; and one can define a filtered chain map $\bar{\Xi}$ as in (6-4), depending on some choices, which induces an isomorphism on spectral sequences from the $E^{2}$ term on. It follows that $\bar{\Xi}$ induces an isomorphism of filtered modules

$$
\bar{\Xi}_{*}: H F_{*}(Z) \stackrel{\simeq}{\longrightarrow} H F_{*}^{\prime}(Z) .
$$


We now want to show that this isomorphism is canonical. For $t=0,1$, let $\left(g_{t}^{Z}, f_{t}^{B}, g_{t}^{B}\right)$ be data to define $\mathcal{C}_{*}$, and let $\mathfrak{C}_{*}^{\prime \prime}(t)$ denote the above subcomplex of $\mathfrak{C}_{*}$. Let $\mathfrak{C}_{*}^{\prime \prime}$ be the intersection $\mathfrak{C}_{*}^{\prime}(0) \cap \mathfrak{C}_{*}^{\prime}(1)$. By the usual argument, the inclusion $\mathfrak{C}_{*}^{\prime \prime} \rightarrow \mathfrak{C}_{*}^{\prime}(t)$ induces an isomorphism on homology. For $t=0,1$, let

$$
\bar{\Xi}_{t}: \mathfrak{C}_{*}^{\prime}(t) \longrightarrow \mathcal{C}_{*}\left(Z, g_{t}^{Z}, f_{t}^{B}, g_{t}^{B}\right)
$$

be a choice of filtered chain map as in (6-4). To show that the isomorphism (6-11) is canonical, it is enough to show that the diagram

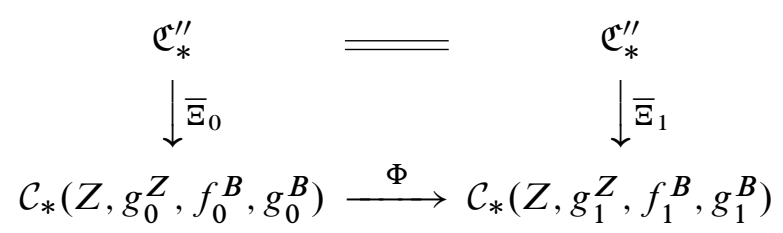

commutes up to filtered chain homotopy, where $\Phi$ is the filtered chain homotopy equivalence given by Proposition 6.4. The proof of this is similar to the proof in Section 6.2 that $\bar{\Xi}$ is a filtered chain map, and is omitted.

\section{Poincaré duality}

The alternate definition of the spectral sequence and family Morse homology described in Section 6, while practical for direct calculations, has some disadvantages. For example, it is hard to generalize it to the case when the base $B$ is noncompact. Moreover it seems difficult, using this definition alone, to prove the naturality property, or to even state the Mayer-Vietoris property. However, one advantage of the alternate definition is that it makes it easy to prove the Poincare duality properties (e) and $\left(\mathrm{e}^{\prime}\right)$ of the Main Principle, as we now explain.

\subsection{Poincaré duality for a single Morse function}

Morse homology has a dual notion of Morse cohomology which counts flow lines going in the other direction, and which is obtained by algebraically dualizing the chain complex:

$$
C_{\text {Morse }}^{*}(f, g):=\operatorname{Hom}\left(C_{*}^{\text {Morse }}(f, g), \mathbb{Z}\right) .
$$

A generic homotopy from $\left(f_{0}, g_{0}\right)$ to $\left(f_{1}, g_{1}\right)$ induces a continuation map

$$
\Phi: C_{\text {Morse }}^{*}\left(f_{0}, g_{0}\right) \longrightarrow C_{\text {Morse }}^{*}\left(f_{1}, g_{1}\right)
$$

which is the dual of the continuation map $C_{*}^{\text {Morse }}\left(f_{1}, g_{1}\right) \rightarrow C_{*}^{\text {Morse }}\left(f_{0}, g_{0}\right)$ induced by the reverse homotopy. 
On an oriented manifold $X$, a coherent orientation $\mathfrak{o}$ for $f$ determines a coherent orientation for $-f$ which we denote by $-\mathfrak{o}$, so in this case we can dualize the Morse complex in another way by defining

$$
\widehat{C}_{*}^{\text {Morse }}:=C_{*}^{\text {Morse }}(-f, g,-\mathfrak{o}) \text {. }
$$

The orientations work out so that the obvious identification

$$
\widehat{C}_{*}^{\text {Morse }}=C_{\text {Morse }}^{\operatorname{dim}(X)-*}(f, g, \mathfrak{o})
$$

is an isomorphism of chain complexes. Since $f$ is homotopic to $-f$, this implies classical Poincaré duality.

\subsection{Poincaré duality for a family of functions}

Given a family $Z=(\pi, f, \mathfrak{o}, \nabla)$ as in Section 3.1, we now have two ways to dualize the associated spectral sequence $E_{*, *}^{*}(Z)$. First, we can simply take the algebraic dual of everything to obtain a cohomological spectral sequence $E_{*}^{*, *}$ with

$$
E_{2}^{i, j}=H^{i}\left(B ; \mathcal{F}^{j}(Z)\right) .
$$

This satisfies dual versions of all the properties of the spectral sequence $E_{*, *}^{*}$.

Second, if the fibers of the family are oriented, then the spectral sequence for the dual family $(\pi,-f,-\mathfrak{o}, \nabla)$ gives a homological spectral sequence, which we denote by $\widehat{E}_{*, *}^{*}$, with

$$
\widehat{E}_{i, j}^{2}=H_{i}\left(B ; \widehat{\mathcal{F}}_{j}(Z)\right) .
$$

Let $n$ denote the dimension of the fibers. If $b \in B$, then Poincare duality for the Morse theory on the fiber over $b$ gives a canonical isomorphism of stalks $\widehat{\mathcal{F}}_{j}(Z)_{b}=$ $\mathcal{F}^{n-j}(Z)_{b}$. If $Z$ is to be regarded as a family of oriented manifolds, then it is natural to assume that the fibers are compatibly oriented, ie that the orientation of the fiber $Z_{b}$ depends continuously on $b$. Under this assumption, these isomorphisms of stalks depend continuously on $b$, so that we have a canonical isomorphism of local coefficient systems $\widehat{\mathcal{F}}_{j}(Z)=\mathcal{F}^{n-j}(Z)$. If the base $B$ is also a closed oriented $m$-manifold, then Poincaré duality on $B$ with local coefficients gives a canonical isomorphism

$$
E_{2}^{i, j}=\widehat{E}_{m-i, n-j}^{2} .
$$

Proposition 7.1 (Poincaré duality) If the base and fibers of the family $Z$ are closed oriented $m$ - and $n$-dimensional manifolds, and if the fibers are compatibly oriented, then the isomorphism (7-1) induces a canonical isomorphism of spectral sequences for $k \geq 2$,

$$
E_{k}^{i, j}=\widehat{E}_{m-i, n-j}^{k}
$$


Proof This is transparent using the alternate definition of the spectral sequence from Section 6.1. If we fix Morse data $\left(f^{B}, g^{B}, \mathfrak{o}^{B}\right)$ on $B$ as in Section 6.1, then we have a canonical identification of bigraded chain complexes

$$
\mathcal{C}^{i, j}\left(Z, g^{Z}, f^{B}, g^{B}, \mathfrak{o}^{B}\right)=\widehat{\mathcal{C}}_{m-i, n-j}\left(Z, g^{Z},-f^{B}, g^{B},-\mathfrak{o}^{B}\right) .
$$

Here $\mathcal{C}^{*, *}$ denotes the algebraic dual of $\mathcal{C}_{*, *}$, and $\widehat{\mathcal{C}}_{*, *}$ denotes the chain complex $\mathcal{C}_{*, *}$ in which the fiberwise Morse functions are negated. To see why (7-2) holds, note that each chain complex can be regarded as generated by pairs $(x, p)$ where $x$ is an index $i$ critical point of $f^{B}$ and $p$ is an index $j$ critical point of $f_{x}$. The differential in the first chain complex counts flow lines of the vector field $\mathcal{V}$ in Equation (6-1), while the differential in the second chain complex counts flow lines of $-\mathcal{V}$, and these are equivalent. Our orientation assumptions ensure that the flow lines are counted with the same signs in both chain complexes.

By (7-2) we have a canonical identification of spectral sequences

$$
\mathcal{E}_{k}^{i, j}\left(Z, g^{Z}, f^{B}, g^{B}, \mathfrak{o}^{B}\right)=\widehat{\mathcal{E}}_{m-i, n-j}^{k}\left(Z, g^{Z},-f^{B}, g^{B},-\mathfrak{o}^{B}\right) .
$$

By Proposition 6.1 we have canonical isomorphisms of spectral sequences $\mathcal{E}_{*}^{*, *}=E_{*}^{*, *}$ and $\widehat{\mathcal{E}}_{*, *}^{*}=\widehat{E}_{*, *}^{*}$ from the second term on. When $k=2$, the isomorphism (7-3) is Poincaré duality in the base and fibers simultaneously and thus agrees with (7-1).

We can now give an a priori proof of property $\left(\mathrm{e}^{\prime}\right)$ in the Main Principle.

Proposition 7.2 Under the assumptions of Proposition 7.1, there is a canonical isomorphism

$$
F_{i} H F_{j}(Z)=F^{m-i} H F^{m+n-j}(Z) .
$$

Proof It is immediate from (7-2) that there is an isomorphism

$$
F_{i} H F_{j}^{\prime}(Z) \simeq F^{m-i} H{F^{\prime}}^{m+n-j}\left(Z^{\vee}\right) .
$$

Furthermore this isomorphism is canonical, ie it commutes with the isomorphisms given by Proposition 6.4. The result now follows from Proposition 6.5 and its dual analogue.

\section{Filtered chain homotopy type}

The spectral sequence and family Morse homology that we have studied so far are derived from a filtered chain complex $\mathfrak{C}_{*}$, defined using singular homology on the base. We now refine the above results by showing that the filtered chain homotopy type of $\mathfrak{C}_{*}$ satisfies the homotopy invariance and naturality properties $\left(\mathrm{b}^{\prime}\right)$ and $\left(\mathrm{c}^{\prime}\right)$ in the Main Principle. 


\subsection{Using a smaller complex of singular cubes}

To prepare for the proof of homotopy invariance and naturality, this subsection proves a result (Lemma 8.5 below) which allows one in the construction of $\mathfrak{C}_{*}$ to use a smaller complex of singular cubes on $B$, without affecting the filtered chain homotopy type.

Definition 8.1 A chain map $j: C_{*}^{\prime} \rightarrow C_{*}$ is a deformation retract if there is a chain map $\psi: C_{*} \rightarrow C_{*}^{\prime}$ and a module homomorphism $L: C_{*} \rightarrow C_{*+1}$ such that

$$
\begin{aligned}
\psi j & =\mathbb{1}, \\
\partial L+L \partial & =\mathbb{1}-j \psi .
\end{aligned}
$$

If $C_{*}$ and $C_{*}^{\prime}$ are filtered, a filtered chain map $j: C_{*}^{\prime} \rightarrow C_{*}$ is a filtered deformation retract if there exist $\psi$ and $L$ as above that are filtered in the sense of Definition 1.6 (ie $\psi$ respects the filtration and $L$ increases the filtration by at most 1 ).

Definition 8.2 If $(\sigma, g)$ is an admissible pair as in Definition 3.3, let $\mathfrak{C}_{*}(\sigma, g)$ denote the filtered complex generated by triples $\left(\sigma^{\prime}, g_{\sigma^{\prime}}, p\right)$ where $\sigma^{\prime}$ is a face of $\sigma$ and $p \in \operatorname{Crit}\left(\sigma^{\prime}(0)\right)$, with the differential $\delta$. Here we do not mod out by degenerate triples. Thus the obvious map $\mathfrak{C}_{*}(\sigma, g) \rightarrow \mathfrak{C}_{*}$ is an inclusion provided that $\left(\sigma^{\prime}, g_{\sigma^{\prime}}\right)$ is nondegenerate for each face $\sigma^{\prime}$ of $\sigma$.

Lemma 8.3 Let $(\sigma, g)$ be an admissible pair, and let $\sigma^{\prime}$ be a face of $\sigma$. Then the inclusion

$$
j: \mathfrak{C}_{*}\left(\sigma^{\prime}, g_{\sigma^{\prime}}\right) \longrightarrow \mathfrak{C}_{*}(\sigma, g)
$$

is a filtered deformation retract.

Proof Without loss of generality, $\sigma^{\prime}$ is the restriction of $\sigma:[-1,1]^{i} \rightarrow B$ to $[-1,1]^{i^{\prime}} \times$ $\{(1, \ldots, 1)\}$. We now proceed in three steps.

Step 1 Let $\mathfrak{p}:[-1,1]^{i} \rightarrow[-1,1]^{i^{\prime}} \times\{(1, \ldots, 1)\}$ denote the projection sending the point $\left(x_{1}, \ldots, x_{i}\right)$ to $\left(x_{1}, \ldots, x_{i^{\prime}}, 1, \ldots, 1\right)$. Consider the degenerate cube

$$
\bar{\sigma}:=\sigma^{\prime} \circ \mathfrak{p}:[-1,1]^{i} \longrightarrow B .
$$

Let $\bar{g}$ denote the fiberwise metric for $\bar{\sigma}$ obtained by pulling back the fiberwise metric $g_{\sigma^{\prime}}$ for $\sigma^{\prime}$ via $\mathfrak{p}$. Similarly to the proof of Proposition 4.5, there is a canonical isomorphism of chain complexes

$$
\mathfrak{C}_{*}(\bar{\sigma}, \bar{g})=\mathfrak{C}_{*}\left(\sigma^{\prime}, g_{\sigma^{\prime}}\right) \otimes C_{*}^{\text {cell }}\left([-1,1]^{i-i^{\prime}}\right) .
$$


Here $C_{*}^{\text {cell }}\left([-1,1]^{i-i^{\prime}}\right)$ denotes the cellular chain complex for the cell decomposition of $[-1,1]^{i-i^{\prime}}$ given by its faces. Now regard $\sigma^{\prime}$ as the face of $\bar{\sigma}$ given by the restriction of $\bar{\sigma}$ to $[-1,1]^{i^{\prime}} \times\{(1, \ldots, 1)\}$. This defines an inclusion

$$
\bar{j}: \mathfrak{C}_{*}\left(\sigma^{\prime}, g_{\sigma^{\prime}}\right) \longrightarrow \mathfrak{C}_{*}(\bar{\sigma}, \bar{g}) .
$$

It follows immediately from $(8-1)$ that $\bar{j}$ is a filtered deformation retract. Thus we can choose a filtered chain map $\bar{\psi}: \mathfrak{C}_{*}(\bar{\sigma}, \bar{g}) \rightarrow \mathfrak{C}_{*}\left(\sigma^{\prime}, g_{\sigma^{\prime}}\right)$ and a filtered chain homotopy $\bar{L}: \mathfrak{C}_{*}(\bar{\sigma}, \bar{g}) \rightarrow \mathfrak{C}_{*+1}(\bar{\sigma}, \bar{g})$ satisfying

$$
\begin{aligned}
\bar{\psi} \bar{j} & =\mathbb{1}, \\
\delta \bar{L}+\bar{L} \delta & =\mathbb{1}-\bar{j} \bar{\psi} .
\end{aligned}
$$

Step 2 Consider a family $\widetilde{Z} \rightarrow[0,1] \times[-1,1]^{i}$ such that

$$
\left.\widetilde{Z}\right|_{\{0\} \times[-1,1]^{i}}=\sigma^{*} Z \text { and }\left.\widetilde{Z}\right|_{\{1\} \times[-1,1]^{i}}=\bar{\sigma}^{*} Z,
$$

while

$$
\left.\widetilde{Z}\right|_{\{t\} \times[-1,1]^{i^{\prime} \times\{(1, \ldots, 1)\}}}=\left(\sigma^{\prime}\right)^{*} Z
$$

for each $t \in[0,1]$. From this family, the construction in the proof of Proposition 6.4 defines a filtered chain map

$$
\Phi: \mathfrak{C}_{*}(\sigma, g) \longrightarrow \mathfrak{C}_{*}(\bar{\sigma}, \bar{g}) .
$$

Similarly, we can define a filtered chain map

$$
\bar{\Phi}: \mathfrak{C}_{*}(\bar{\sigma}, \bar{g}) \longrightarrow \mathfrak{C}_{*}(\sigma, g)
$$

and a filtered chain homotopy

$$
\begin{gathered}
K: \mathfrak{C}_{*}(\sigma, g) \longrightarrow \mathfrak{C}_{*+1}(\sigma, g), \\
\delta K+K \delta=\mathbb{1}-\bar{\Phi} \Phi .
\end{gathered}
$$

In the construction of $\Phi$, we can use the standard Morse function (3-4) and the Euclidean metric on $\{t\} \times[-1,1]^{i}$ for each $t \in[0,1]$, and we can choose the fiberwise metric on $\bar{Z}$ to agree with $g_{\sigma^{\prime}}$ on $\{t\} \times[-1,1]^{i^{\prime}} \times\{(1, \ldots, 1)\}$ for each $t \in[0,1]$. With such choices,

$$
\Phi j=\bar{j}
$$

We can similarly arrange that

$$
\bar{\Phi} \bar{j}=j .
$$


Step 3 To complete the proof of the lemma, define

$$
\begin{aligned}
\psi & :=\bar{\psi} \Phi: \mathfrak{C}_{*}(\sigma, g) \longrightarrow \mathfrak{C}_{*}\left(\sigma^{\prime}, g_{\sigma^{\prime}}\right), \\
L & :=K+\bar{\Phi} \bar{L} \Phi: \mathfrak{C}_{*}(\sigma, g) \longrightarrow \mathfrak{C}_{*+1}(\sigma, g) .
\end{aligned}
$$

We now check that $\psi$ and $L$ have the required properties. It follows from (8-5) and (8-2) that $\psi j=\mathbb{1}$. It follows from (8-4), (8-3), and (8-6) that $\delta L+L \delta=\mathbb{1}-j \psi$. Finally, $\psi$ and $L$ are filtered because their constituents are.

Let $C_{*}^{\text {adm }}(B)$ denote the subcomplex of the cubical singular chain complex of $B$ generated by admissible cubes in $B$; see Definition 3.3. Suppose $S$ is a set of admissible cubes in $B$, such that every face of a cube in $S$ is also in $S$. Let $C_{*}^{\prime}$ denote the subcomplex of $C_{*}^{\mathrm{adm}}(B)$ generated by cubes in $S$. Cubes in $S$ will be called "generators of $C_{*}^{\prime}$ ", even though degenerate cubes in $S$ actually represent zero in $C_{*}^{\prime}$. We now consider a situation where the inclusion $j_{0}: C_{*}^{\prime} \rightarrow C_{*}^{\mathrm{adm}}(B)$ is a deformation retract, in which the requisite maps $\psi_{0}: C_{*}^{\operatorname{adm}}(B) \rightarrow C_{*}^{\prime}$ and $L_{0}: C_{*}^{\operatorname{adm}}(B) \rightarrow C_{*+1}^{\operatorname{adm}}(B)$ can be chosen to have a particularly nice form, sending individual cubes to individual cubes.

Definition 8.4 The inclusion $j_{0}: C_{*}^{\prime} \rightarrow C_{*}^{\mathrm{adm}}(B)$ is a cubical deformation retract if for each admissible (possibly degenerate) cube $\sigma:[-1,1]^{i} \rightarrow B$, one can choose a cube $L_{0}(\sigma):[-1,1]^{i+1} \rightarrow B$, such that:

- $\left.L_{0}(\sigma)\right|_{\{1\} \times[-1,1]^{i}}=\sigma$.

- $\psi_{0}(\sigma):=\left.L_{0}(\sigma)\right|_{\{-1\} \times[-1,1]^{i}}$ is a generator of $C_{*}^{\prime}$.

- If $\sigma^{\prime}$ is a face of $\sigma$, then $L_{0}\left(\sigma^{\prime}\right)$ is the face $[-1,1] \times \sigma^{\prime}$ of $L_{0}(\sigma)$.

- If $\sigma$ is a generator of $C_{*}^{\prime}$, then $L_{0}(\sigma)$ is the degenerate cube $[-1,1] \times \sigma$.

- If $\sigma$ is independent of the $j$-th coordinate on $[-1,1]^{i}$, then $L_{0}(\sigma)$ is independent of the $(j+1)$-st coordinate on $[-1,1]^{i+1}$.

For $C_{*}^{\prime}$ as above, let $\mathfrak{C}_{*}^{\prime}$ denote the subcomplex of $\mathfrak{C}_{*}^{*}$ generated by triples $(\sigma, g, p)$ where $\sigma$ is a generator of $C_{*}^{\prime}$.

Lemma 8.5 Suppose that the inclusion $j_{0}: C_{*}^{\prime} \rightarrow C_{*}^{\operatorname{adm}}(B)$ is a cubical deformation retract. Then the inclusion $J: \mathfrak{C}_{*}^{\prime} \rightarrow \mathfrak{C}_{*}$ is a filtered deformation retract.

Proof We will construct a filtered chain map $\Psi: \mathfrak{C}_{*} \rightarrow \mathfrak{C}_{*}^{\prime}$ and a filtered chain homotopy $\mathfrak{L}: \mathfrak{C}_{*} \rightarrow \mathfrak{C}_{*+1}$ that "lift" $\psi_{0}$ and $L_{0}$ as follows. For each (possibly degenerate) admissible pair $(\sigma, g)$, extend $g$ to a generic fiberwise metric $\bar{g}$ over $L_{0}(\sigma)$. Choose these metrics by induction on the dimension of $\sigma$ so that: 
(i) If $\sigma^{\prime}$ is a face of $\sigma$, then $\left.\bar{g}\right|_{\sigma^{\prime}}=\overline{g_{\sigma^{\prime}}}$.

(ii) If $\sigma$ is a generator of $C_{*}^{\prime}$, so that $L_{0}(\sigma)$ is the degenerate cube $[-1,1] \times \sigma$, then $\bar{g}$ is pulled back from $g$.

(iii) If $(\sigma, g)$ is independent of the $j$-th coordinate on $[-1,1]^{i}$, then $\bar{g}$ is independent of the $(j+1)$-st coordinate on $[-1,1]^{i+1}$.

For each admissible pair $(\sigma, g)$, we now define maps

$$
\begin{aligned}
& \mathfrak{L}_{(\sigma, g)}: \mathfrak{C}_{*}(\sigma, g) \longrightarrow \mathfrak{C}_{*+1}\left(L_{0}(\sigma), \bar{g}\right), \\
& \Psi_{(\sigma, g)}: \mathfrak{C}_{*}(\sigma, g) \longrightarrow \mathfrak{C}_{*}\left(\psi_{0}(\sigma), \bar{g}_{\psi_{0}(\sigma)}\right),
\end{aligned}
$$

which will fit together to give the maps $\mathfrak{L}$ and $\Psi$.

To define the maps (8-7), fix an admissible pair $(\sigma, g)$, and let

$$
j: \mathfrak{C}_{*}\left(\psi_{0}(\sigma), \bar{g}_{\psi_{0}(\sigma)}\right) \longrightarrow \mathfrak{C}_{*}\left(L_{0}(\sigma), \bar{g}\right)
$$

denote the inclusion. By Lemma 8.3, there exist a filtered chain map

$$
\psi: \mathfrak{C}_{*}\left(L_{0}(\sigma), \bar{g}\right) \longrightarrow \mathfrak{C}_{*}\left(\psi_{0}(\sigma), \bar{g}_{\psi_{0}(\sigma)}\right),
$$

and a filtered chain homotopy

$$
L: \mathfrak{C}_{*}\left(L_{0}(\sigma), \bar{g}\right) \longrightarrow \mathfrak{C}_{*+1}\left(L_{0}(\sigma), \bar{g}\right),
$$

such that

$$
\delta L+L \delta=\mathbb{1}-j \psi
$$

on $\mathfrak{C}_{*}\left(L_{0}(\sigma), \bar{g}\right)$. By conditions (i)-(iii) above and the proof of Lemma 8.3, we can choose these maps for each admissible pair $(\sigma, g)$ by induction on the dimension of $\sigma$ so that:

$\left(i^{\prime}\right)$ If $\sigma^{\prime}$ is a face of $\sigma$ such that the pair $\left(\sigma^{\prime}, g_{\sigma^{\prime}}\right)$ is nondegenerate, then the maps $\psi$ and $L$ for $\left(\sigma^{\prime}, g^{\prime}\right)$ are the restrictions of those for $(\sigma, g)$.

(ii') If $\sigma$ is a generator of $C_{*}^{\prime}$, then $\left.\psi\right|_{\mathfrak{C}_{*}(\sigma, g)}$ is the tautological identification

$$
\mathfrak{C}_{*}(\sigma, g)=\mathfrak{C}_{*}\left(\psi_{0}(\sigma), \bar{g}_{\psi_{0}(\sigma)}\right) .
$$

(iii $\left.{ }^{\prime}\right)$ If $\sigma^{\prime}$ is a face of $\sigma$ such that the pair $\left(\sigma^{\prime}, g_{\sigma^{\prime}}\right)$ is degenerate, then $\psi$ and $L$ send every element of $\mathfrak{C}_{*}\left(\sigma^{\prime}, g_{\sigma^{\prime}}\right)$ to a linear combination of degenerate triples.

Now define the maps (8-7) by

$$
\begin{aligned}
& \Psi_{(\sigma, g)}:=\left.\psi\right|_{\mathfrak{C}_{*}(\sigma, g)}, \\
& \mathfrak{L}_{(\sigma, g)}:=\left.L\right|_{\mathfrak{C}_{*}(\sigma, g)} .
\end{aligned}
$$

Algebraic 83 Geometric Topology, Volume 8 (2008) 
By conditions ( $\left.\mathrm{i}^{\prime}\right)$ and (iii'), these maps for the different pairs $(\sigma, g)$ fit together to give a well-defined filtered chain map $\Psi: \mathfrak{C}_{*} \rightarrow \mathfrak{C}_{*}^{\prime}$, and a well-defined map $\mathfrak{L}: \mathfrak{C}_{*} \rightarrow \mathfrak{C}_{*+1}$ which increases the filtration by at most 1 . Then Equation (8-8) implies the chain homotopy property $\delta \mathfrak{L}+\mathfrak{L} \delta=\mathbb{1}-J \Psi$. Finally, condition (ii') implies that $\Psi J=\mathbb{1}$ on $\mathfrak{C}_{*}^{\prime \prime}$.

\subsection{Homotopy invariance and naturality}

We now prove the homotopy invariance property $\left(\mathrm{b}^{\prime}\right)$ in the Main Principle. Below, the notation " $\phi_{0} \sim \phi_{1}$ " indicates that $\phi_{0}$ is filtered chain homotopic to $\phi_{1}$.

Proposition 8.6 (Homotopy invariance) Let $(\pi: Z \rightarrow[0,1] \times B, f, \nabla)$ be a family as in Section 3.1 such that $Z_{0}:=\left.Z\right|_{\{0\} \times B}$ and $Z_{1}:=\left.Z\right|_{\{1\} \times B}$ are admissible. Then there is a filtered chain homotopy equivalence $\Phi(Z): \mathfrak{C}_{*}\left(Z_{0}\right) \rightarrow \mathfrak{C}_{*}\left(Z_{1}\right)$, which is well-defined up to filtered chain homotopy, such that:

(i) Let $\pi: \bar{Z} \rightarrow[0,1]^{2} \times B$ be a family where $\left.\bar{Z}\right|_{[0,1] \times\{0\} \times B}$ and $\left.\bar{Z}\right|_{[0,1] \times\{1\} \times B}$ are pulled back from $Z_{0}$ and $Z_{1}$ respectively. Then

$$
\Phi\left(\left.\bar{Z}\right|_{\{0\} \times[0,1] \times B}\right) \sim \Phi\left(\left.\bar{Z}\right|_{\{1\} \times[0,1] \times B}\right) .
$$

(ii) Let $\widehat{Z}$ be a family over $[0,2] \times B$ such that $Z_{t}:=\left.Z\right|_{\{t\} \times B}$ is admissible for $t=0,1,2$. Then

$$
\Phi(\widehat{Z}) \sim \Phi\left(\left.\widehat{Z}\right|_{[1,2] \times B}\right) \circ \Phi\left(\left.\widehat{Z}\right|_{[0,1] \times B}\right) .
$$

(iii) If $Z$ is pulled back via the projection $[0,1] \times B \rightarrow B$ from a family $Z_{0} \rightarrow B$, then $\Phi(Z) \sim \mathbb{1}$.

(iv) $\Phi(Z)$ induces the isomorphism on spectral sequences (1-5).

Proof There are three steps.

Step 1 Let $C_{*}^{\prime}$ denote the subcomplex of $C_{*}^{\operatorname{adm}}(B)$ generated by singular cubes that are admissible for both of the families $Z_{0}$ and $Z_{1}$. Since any smooth cube in $B$ can be perturbed so as to be admissible for both families, it follows that the inclusion of $C_{*}^{\prime}$ into the complex generated by cubes that are admissible for just one of the families is a cubical deformation retract. Now the subcomplex $C_{*}^{\prime}$ determines subcomplexes $\mathfrak{C}_{*}^{\prime \prime}\left(Z_{0}\right)$ and $\mathfrak{C}_{*}^{\prime \prime}\left(Z_{1}\right)$ of $\mathfrak{C}_{*}\left(Z_{0}\right)$ and $\mathfrak{C}_{*}\left(Z_{1}\right)$ respectively. By Lemma 8.5 , these subcomplexes are filtered deformation retracts, and so it suffices to define a filtered chain map

$$
\Phi: \mathfrak{C}_{*}^{\prime}\left(Z_{0}\right) \longrightarrow \mathfrak{C}_{*}^{\prime \prime}\left(Z_{1}\right)
$$


with the desired properties.

Step 2 We now define a map (8-9), similarly to the proofs of Proposition 6.4 and Lemma 8.3. Suppose $\sigma:[-1,1]^{i} \rightarrow B$ is a cube which is admissible for both $Z_{0}$ and $Z_{1}$, and suppose $g$ is a fiberwise metric on $\sigma^{*} Z_{0}$ such that $(\sigma, g)$ is admissible for $Z_{0}$. Let

$$
\bar{\sigma}:=\operatorname{id} \times \sigma:[0,1] \times[-1,1]^{i} \longrightarrow[0,1] \times B .
$$

Choose a generic fiberwise metric $\bar{g}$ on $\bar{\sigma}^{*} Z$ such that $\left.\bar{g}\right|_{\{0\} \times[-1,1]^{i}}=g$ and such that $\left(\sigma,\left.\bar{g}\right|_{\{1\} \times[-1,1]^{i}}\right)$ is admissible for $Z_{1}$. Choose the metrics $\bar{g}$ for each pair $(\sigma, g)$ as above by induction on $i$ so that they are compatible with face maps and degeneracies, as in conditions (i) and (iii) in the proof of Lemma 8.5. For $t \in[0,1]$, let $\xi_{t}$ denote the fiberwise negative gradient of $f$ with respect to $\bar{g}$ over $\{t\} \times B$. By analogy with (6-9), define a vector field $\bar{V}$ on $\bar{\sigma}^{*} Z$ as follows: If $t \in[0,1], x \in[-1,1]^{i}$, and $y \in Z_{(t, x)}$, then

$$
\bar{V}(t, x, y):=\beta(t) \partial_{t}+\xi_{t}+H_{t} W_{i} .
$$

Here $W_{i}$ is the standard vector field on the cube $[-1,1]^{i}$ defined in (3-3). We now define $\Phi$ by counting flow lines of the vector field $\bar{V}$. The proof of Proposition 6.4, with $B$ replaced by $[-1,1]^{i}$, shows that $\Phi$ is a filtered chain map which is well-defined up to filtered chain homotopy and satisfies conditions (i)-(iii). In particular, it follows that $\Phi$ is a filtered chain homotopy equivalence.

Step 3 We now prove property (iv). It is enough to show that $\Phi$ induces the correct map on the $E^{2}$ terms of the spectral sequences. We proceed similarly to the proof of Proposition 4.1. For $t=0,1$, let $\widetilde{B}_{t}$ denote the space of pairs $(b, g)$, where $b \in B$ and $g$ is a metric on $Z_{(t, b)}$. As in (4-2), for $t=0,1$ the $E^{1}$ terms of the spectral sequences are given by

$$
E_{i, j}^{1}\left(\mathfrak{C}_{*}^{\prime}\left(Z_{t}\right)\right)=C_{i}^{\prime}\left(\widetilde{B}_{t} ; \mathcal{F}_{j}\left(Z_{t}\right)\right)
$$

Here $C_{i}^{\prime}$ denotes the complex of singular cubes in $\widetilde{B}_{t}$ corresponding to pairs $(\sigma, g)$, where $\sigma$ is admissible for both $Z_{0}$ and $Z_{1}$, and $(\sigma, g)$ is admissible for $Z_{t}$.

Now let $\phi: \mathcal{F}_{*}\left(Z_{0}\right) \rightarrow \mathcal{F}_{*}\left(Z_{1}\right)$ denote the isomorphism of local coefficient systems defined by continuation along paths $[0,1] \times\{b\}$ for $b \in B$. It follows directly from the definition of $\Phi$ in Step 2 that the induced map on $E^{1}$ terms fits into a commutative diagram

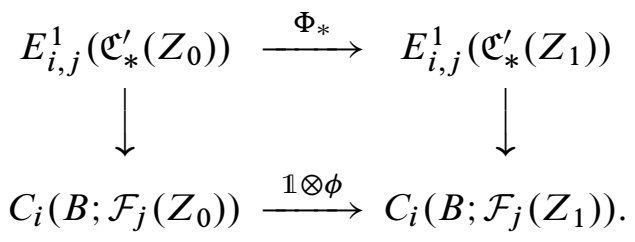


Here the vertical arrows are defined using the identification (8-10) followed by projection from $\widetilde{B}_{t}$ to $B$. As in the proof of Proposition 4.1, the vertical arrows induce on homology the canonical isomorphisms

$$
E_{i, j}^{2}\left(Z_{t}\right)=H_{i}\left(B ; \mathcal{F}_{j}\left(Z_{t}\right)\right) .
$$

It follows that $\Phi$ induces the map $\mathbb{1} \otimes \phi$ on the $E^{2}$ terms, as claimed.

We now prove the naturality property $\left(\mathrm{c}^{\prime}\right)$ in the Main Principle.

Proposition 8.7 (Naturality) Let $Z$ be an admissible family over $B$, and $\phi: B^{\prime} \rightarrow B$ a generic smooth map so that $\phi^{*} Z$ is admissible over $B^{\prime}$. Then there is a canonical filtered chain map

$$
\phi_{*}: \mathfrak{C}_{*}\left(\phi^{*} Z\right) \longrightarrow \mathfrak{C}_{*}(Z)
$$

such that:

(i) $\phi_{*}$ induces the map on spectral sequences (4-4).

(ii) (Functoriality) If $\phi^{\prime}: B^{\prime \prime} \rightarrow B^{\prime}$ is a generic smooth map so that the family $\left(\phi \circ \phi^{\prime}\right)^{*} Z$ is admissible over $B^{\prime \prime}$, then

$$
\left(\phi \circ \phi^{\prime}\right)_{*}=\phi_{*} \phi_{*}^{\prime} \text {. }
$$

(iii) (Homotopy invariance) Let $\phi:[0,1] \times B^{\prime} \rightarrow B$, write $\phi_{t}:=\left.\phi\right|_{\{t\} \times B^{\prime}}$, and assume that $\phi_{0}^{*} Z$ and $\phi_{1}^{*} Z$ are admissible. Then the following diagram commutes up to filtered chain homotopy:

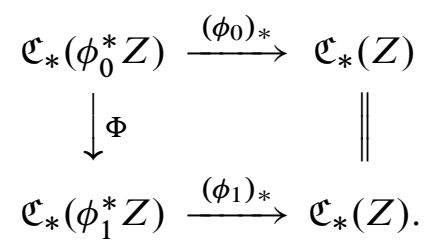

Here $\Phi:=\Phi\left(\phi^{*} Z\right)$ is the filtered chain homotopy equivalence given by Proposition 8.6.

Proof Recall from the proof of Proposition 4.3 that the map on spectral sequences (4-4) is induced by a filtered chain map $\phi_{*}: \mathfrak{C}_{*}\left(\phi^{*} Z\right) \rightarrow \mathfrak{C}_{*}(Z)$ defined by

$$
\phi_{*}(\sigma, g, p):=(\phi \circ \sigma, g, p) .
$$

This is clearly functorial, so we just need to prove that it is homotopy invariant.

Let $\phi$ as in (iii) be given. For notational convenience, reparametrize the interval to regard $\phi$ as a map $[-1,1] \times B^{\prime} \rightarrow B$. Let $C_{*}^{\prime}$ denote the complex generated by singular 
cubes in $B^{\prime}$ that are admissible for both $\phi_{-1}^{*} Z$ and $\phi_{1}^{*} Z$. By Lemma 8.5, the inclusions $\mathfrak{C}_{*}^{\prime}\left(\phi_{-1}^{*} Z\right) \rightarrow \mathfrak{C}_{*}\left(\phi_{-1}^{*} Z\right)$ and $\mathfrak{C}_{*}^{\prime \prime}\left(\phi_{1}^{*} Z\right) \rightarrow \mathfrak{C}_{*}\left(\phi_{1}^{*} Z\right)$ are filtered deformation retracts. Thus it is enough to show that the diagram

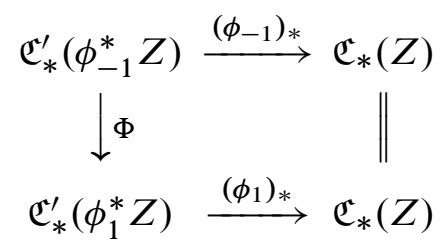

commutes up to filtered chain homotopy.

If $\sigma:[-1,1]^{i} \rightarrow B^{\prime}$ is a generator of $C_{*}^{\prime}$, let

$$
\bar{\sigma}:=\mathrm{id} \times \sigma:[-1,1] \times[-1,1]^{i} \rightarrow[-1,1] \times B^{\prime} .
$$

By perturbing the $\bar{\sigma}$ 's, we may arrange that the $\bar{\sigma}$ 's are admissible for $\phi^{*} Z$. Now for each fiberwise metric $g$ for $\sigma^{*} \phi_{-1}^{*} Z$ such that the pair $(\sigma, g)$ is admissible for $\phi_{-1}^{*} Z$, choose a fiberwise metric $\bar{g}$ on $\bar{\sigma}^{*} Z$ such that the pair $(\bar{\sigma}, \bar{g})$ is admissible for $\bar{\sigma}^{*} Z$. Choose these perturbations and metrics to be compatible with face maps and degeneracies, as in conditions (i) and (iii) in the proof of Lemma 8.5.

By Lemma 8.3, for each pair $(\sigma, g)$ as above we can choose a filtered chain map and a filtered chain homotopy

$$
\begin{aligned}
\Psi_{(\sigma, g)}: & \mathfrak{C}_{*}(\{-1\} \times \sigma, g) \longrightarrow \mathfrak{C}_{*}\left(\{1\} \times \sigma, \bar{g}_{\{1\} \times \sigma}\right), \\
\mathfrak{L}_{\sigma, g}: & \mathfrak{C}_{*}(\{-1\} \times \sigma, g) \longrightarrow \mathfrak{C}_{*+1}(\bar{\sigma}, \bar{g})
\end{aligned}
$$

such that if

$$
j: \mathfrak{C}_{*}\left(\{1\} \times \sigma, \bar{g}_{\{1\} \times \sigma}\right) \longrightarrow \mathfrak{C}_{*}(\bar{\sigma}, \bar{g})
$$

denotes the inclusion, then

$$
\delta \mathfrak{L}_{(\sigma, g)}+\mathfrak{L}_{(\sigma, g)} \delta=\mathbb{1}-j \Psi_{(\sigma, g)}
$$

on $\mathfrak{C}_{*}(\{-1\} \times \sigma, g)$. Moreover, as in the proof of Lemma 8.5, the maps $\Psi_{(\sigma, g)}$ and $\mathfrak{L}_{(\sigma, g)}$ for the different $(\sigma, g)$ 's can be chosen to fit together to well-defined maps

$$
\begin{aligned}
& \Psi: \mathfrak{C}_{*}^{\prime}\left(\phi_{-1}^{*} Z\right) \longrightarrow \mathfrak{C}_{*}^{\prime}\left(\phi_{1}^{*} Z\right), \\
& \mathfrak{L}: \mathfrak{C}_{*}\left(\phi_{-1}^{*} Z\right) \longrightarrow \mathfrak{C}_{*+1}\left(\phi^{*} Z\right)
\end{aligned}
$$

so that if

$$
J: \mathfrak{C}_{*}^{\prime}\left(\phi_{1}^{*} Z\right) \longrightarrow \mathfrak{C}_{*}\left(\phi^{*} Z\right)
$$

denotes the inclusion, then on $\mathfrak{C}_{*}^{\prime}\left(\phi_{-1}^{*} Z\right)$,

$$
\delta \mathfrak{L}+\mathfrak{L} \delta=\mathbb{1}-J \Psi .
$$


Inspection of the proof of Lemma 8.3 shows that for suitable choices, the map $\Psi$ in (8-11) agrees with the filtered chain homotopy equivalence $\Phi:=\Phi\left(\phi^{*} Z\right)$ defined in Proposition 8.6. Then applying the pushforward $\phi_{*}$ to Equation (8-12) gives

$$
\delta\left(\phi_{*} \mathfrak{L}\right)+\left(\phi_{*} \mathfrak{L}\right) \delta=\left(\phi_{-1}\right)_{*}-\left(\phi_{1}\right)_{*} \Phi
$$

on $\mathfrak{C}_{*}^{\prime \prime}\left(\phi_{-1}^{*} Z\right)$. Thus $\phi_{*} \mathfrak{L}$ is the desired filtered chain homotopy.

\subsection{Concluding remarks}

We conjecture that $\mathfrak{C}_{*}$ and $\mathcal{C}_{*}$ have the same filtered homotopy type. (We have only shown that they determine the same spectral sequence and homology.) More precisely, we expect that the map (6-4) is a filtered chain homotopy equivalence; by Lemma 8.5 , this would imply the conjecture.

We also conjecture that $\mathfrak{C}_{*}$ has the same filtered chain homotopy type as the cubical singular chain complex $C_{*}(Z)$, with the filtration that defines the Leray-Serre spectral sequence. More precisely, we expect that the map (5-1) is a filtered chain homotopy equivalence.

\section{Generalization to Novikov homology}

We now generalize the above constructions to study Novikov homology of families, given a suitable family of closed one-forms on the fibers of a smooth fiber bundle $Z \rightarrow B$. We will see that Novikov homology of families involves some subtleties which do not arise for Morse homology of families.

\subsection{Novikov homology}

We begin with a brief review of Novikov homology. To prepare for the generalization to families, we need to be especially careful about how Novikov homology depends on certain choices.

Let $X$ be a closed, connected smooth manifold. Let $\omega$ be a Morse 1-form on $X$; this means that $d \omega=0$, and locally $\omega$ is $d$ of a Morse function. Let $g$ be a metric on $X$, and let $\xi$ be the vector field dual to $-\omega$ via $g$. We assume that $g$ is generic so that $\xi$ satisfies the Morse-Smale transversality condition. Finally, fix a reference point $x_{0} \in X$. We now define a version of Novikov homology, which we denote by $H_{*}^{\text {Nov }}\left(\omega, g, x_{0}\right)$, as follows. 
The first step is to specify the coefficient ring $\Lambda$ of the chain complex. There are various options for this, but for simplicity we will fix $\Lambda$ as follows. Let

$$
K:=\operatorname{Ker}(\omega) \subset H_{1}(X), \quad \Gamma:=H_{1}(X) / K .
$$

Definition 9.1 The Novikov ring

$$
\Lambda:=\operatorname{Nov}(\Gamma,-\omega ; \mathbb{Z})
$$

is the set of functions $\lambda: \Gamma \rightarrow \mathbb{Z}$ such that for all $R \in \mathbb{R}$, there are only finitely many $A \in \Gamma$ with $\lambda(A) \neq 0$ and $\omega(A)>R$. We denote the function $\lambda$ by the possibly infinite formal sum $\sum_{A \in \Gamma} \lambda(A) e^{A}$. Here $e^{A}$ is a formal symbol. The multiplication rule is defined, as the notation suggests, by $e^{A} e^{B}:=e^{A+B}$. The finiteness condition ensures that the product on $\Lambda$ is well-defined; see eg [20].

If $p, q \in X$, let $H_{1}(X, p, q)$ denote the set of relative homology classes of 1-chains $\eta$ in $X$ with $\partial \eta=p-q$. Observe that $H_{1}(X, p, q)$ is an affine space over $H_{1}(X)$. Likewise $H_{1}(X, p, q) / K$ is an affine space over $\Gamma$.

Definition 9.2 An anchored critical point of $\omega$ is a pair $\tilde{p}=(p, \eta)$ where $p \in X$ is a critical point of $\omega$ and $\eta \in H_{1}\left(X, p, x_{0}\right) / K$. The index of $\tilde{p}$ is defined to be the index of $p$. The action of the anchored critical point $\tilde{p}$ is defined by

$$
\mathcal{A}(\widetilde{p}):=\int_{\eta} \omega \in \mathbb{R} .
$$

Remark 9.3 Let $\pi_{\omega}: \tilde{X}_{\omega} \rightarrow X$ denote the covering space corresponding to the kernel of the composition

$$
\pi_{1}\left(X, x_{0}\right) \longrightarrow H_{1}(X) \stackrel{\omega}{\longrightarrow} \mathbb{R} .
$$

Then an anchored critical point is equivalent to a critical point of the exact 1 -form $\pi_{\omega}^{*} \omega$ on $\tilde{X}_{\omega}$. The latter description is more usual in Novikov theory, but the former description is more convenient for our purposes.

Definition 9.4 The chain module $C_{i}^{\text {Nov }}\left(\omega, g, x_{0}\right)$ is the set of formal sums of index $i$ anchored critical points $\sum_{\tilde{p}} c_{\tilde{p}} \cdot \tilde{p}$ with coefficients $c_{\tilde{p}} \in \mathbb{Z}$, such that for all $R \in \mathbb{R}$, there are only finitely many $\tilde{p}$ with $c_{\tilde{p}} \neq 0$ and $\mathcal{A}(\tilde{p})>R$.

Observe that $C_{i}^{\text {Nov }}\left(\omega, g, x_{0}\right)$ is a free $\Lambda$-module with one generator for each index $i$ critical point of $\omega$. A basis can be specified by choosing, for each such critical point $p$, an "anchor" $\eta_{p} \in H_{1}\left(X, p, x_{0}\right) / K$. 
If $p, q \in X$ are critical points of $\omega$, then a flow line $u$ of $\xi$ from $p$ to $q$ determines a relative homology class $[u] \in H_{1}(X, p, q)$. Given $\mu \in H_{1}(X, p, q) / K$, let $\mathcal{M}(p, q, \mu)$ denote the moduli space of flow lines $u$ of $\xi$ from $p$ to $q$, modulo reparametrization, with $[u]=\mu$. Also, fix orientations of the descending manifolds of the critical points of $\omega$.

Definition 9.5 Define the differential

$$
\text { д: } C_{i}^{\mathrm{Nov}}\left(\omega, g, x_{0}\right) \longrightarrow C_{i-1}^{\mathrm{Nov}}\left(\omega, g, x_{0}\right)
$$

as follows: if $(p, \eta)$ is an index $i$ anchored critical point, then

$$
\partial(p, \eta):=\sum_{q \in \mathrm{Crit}_{i-1}(\omega)} \sum_{\mu \in H_{1}(X, p, q) / K} \# \mathcal{M}(p, q, \mu) \cdot(q, \eta-\mu) .
$$

Standard arguments (see eg Pozniak [33]) show that $\partial$ is well-defined and $\partial^{2}=0$. The homology of this chain complex is the Novikov homology $H_{*}^{\mathrm{Nov}}\left(\omega, g, x_{0}\right)$.

We now consider continuation isomorphisms in Novikov homology. Unlike in Morse homology, these depend on a choice of relative homology class.

Lemma 9.6 (a) $H_{*}^{\text {Nov }}\left(\omega, x_{0}\right):=H_{*}^{\text {Nov }}\left(\omega, g, x_{0}\right)$ does not depend on $g$.

(b) Let $\omega_{0}$ and $\omega_{1}$ be Morse 1-forms on $X$ in the same cohomology class, and let $x_{0}, x_{1} \in X$. A relative homology class $\chi \in H_{1}\left(X, x_{0}, x_{1}\right) / K$ determines a continuation isomorphism

$$
\Phi\left(\omega_{0}, \omega_{1}, \chi\right): H_{*}^{\mathrm{Nov}}\left(\omega_{0}, x_{0}\right) \stackrel{\simeq}{\longrightarrow} H_{*}^{\mathrm{Nov}}\left(\omega_{1}, x_{1}\right)
$$

with the following properties:

(i) If $x_{0}=x_{1}$, then $\phi\left(\omega_{0}, \omega_{0}, 0\right)=\mathbb{1}$ on $H_{*}^{\mathrm{Nov}}\left(\omega_{0}, x_{0}\right)$.

(ii) If $\omega_{2}$ is another Morse 1-form on $X$ in the same cohomology class, if $x_{2} \in X$, and if $\chi_{t} \in H_{1}\left(X, x_{t-1}, x_{t}\right) / K$ for $t=1,2$, then

$$
\Phi\left(\omega_{0}, \omega_{2}, \chi_{1}+\chi_{2}\right)=\Phi\left(\omega_{1}, \omega_{2}, \chi_{2}\right) \circ \Phi\left(\omega_{0}, \omega_{1}, \chi_{1}\right) .
$$

(iii) If $A \in \Gamma$ then

$$
\Phi\left(\omega_{0}, \omega_{1}, \chi+A\right)=e^{A} \Phi\left(\omega_{0}, \omega_{1}, \chi\right) .
$$

Proof Fix Morse 1-forms $\omega_{0}, \omega_{1}$ in the same cohomology class, metrics $g_{0}, g_{1}$ on $X$ such that the pairs $\left(\omega_{0}, g_{0}\right)$ and $\left(\omega_{1}, g_{1}\right)$ are Morse-Smale, reference points $x_{0}, x_{1} \in X$, and a relative homology class $\chi \in H_{1}\left(X, x_{0}, x_{1}\right) / K$. Let $\left\{\omega_{t} \mid t \in[0,1]\right\}$ be a family of closed 1 -forms in the same cohomology class interpolating from $\omega_{0}$ 
to $\omega_{1}$. Let $\left\{g_{t} \mid t \in[0,1]\right\}$ be a generic family of metrics on $X$ interpolating from $g_{0}$ to $g_{1}$.

Given $\mu \in H_{1}\left(X, x_{0}, x_{1}\right) / K$, and given $p_{0} \in \operatorname{Crit}_{i}\left(\omega_{0}\right)$ and $p_{1} \in \operatorname{Crit}_{i}\left(\omega_{1}\right)$, let $\mathcal{M}\left(p_{0}, p_{1}, \mu\right)$ denote the moduli space of flow lines $u$ of the vector field (2-2) on $[0,1] \times X$ from $\left(0, p_{0}\right)$ to $\left(1, p_{1}\right)$, with $[u]=\mu$ under the identification

$$
H_{1}\left(X, x_{0}, x_{1}\right)=H_{1}\left([0,1] \times X,\left(0, x_{0}\right),\left(1, x_{1}\right)\right) .
$$

Define a continuation map

$$
\Phi:=\Phi\left(\left\{\left(\omega_{t}, g_{t}\right)\right\}, \chi\right): C_{*}^{\mathrm{Nov}}\left(\omega_{0}, g_{0}, x_{0}\right) \longrightarrow C_{*}^{\mathrm{Nov}}\left(\omega_{1}, g_{1}, x_{1}\right)
$$

as follows: if $\left(p_{0}, \eta_{0}\right)$ is an index $i$ anchored critical point of $\omega_{0}$, then

$$
\Phi\left(p_{0}, \eta_{0}\right):=\sum_{p_{1} \in \mathrm{Crit}_{i}\left(\omega_{1}\right)} \sum_{\mu \in H_{1}\left(X, x_{0}, x_{1}\right) / K} \# \mathcal{M}\left(p_{0}, p_{1}, \mu\right) \cdot\left(p_{1}, \eta_{0}+\chi-\mu\right) .
$$

Standard arguments show that this is a well-defined chain map with the usual homotopy properties of continuation maps, with dependence on $\chi$ as in (i)-(iii) above. The lemma is a formal consequence of this, with $\Phi\left(\omega_{0}, \omega_{1}, \chi\right)$ defined to be the map induced on homology by $\Phi\left(\left\{\left(\omega_{t}, g_{t}\right)\right\}, \chi\right)$.

\subsection{The sheaf of Novikov homologies}

We now explain how to assemble the Novikov homologies of a family of 1-forms into a local coefficient system over the base. It is here that we encounter subtleties that are not present in the case of family Morse homology.

Let $\pi: Z \rightarrow B$ be a smooth fiber bundle whose fibers are closed manifolds. Let $\omega$ be a family of closed 1 -forms $\omega_{b}$ on $Z_{b}$ for each $b \in B$, depending smoothly on $b$. We assume that the family $\left\{\omega_{b}\right\}$ is "admissible" in the following sense:

Definition 9.7 The family $\omega=\left\{\omega_{b}\right\}$ is admissible if:

- The fibers of $Z \rightarrow B$ are connected.

- The cohomology classes of the $\omega_{b}$ 's describe a locally constant section of the flat vector bundle $\left\{H^{1}\left(Z_{b} ; \mathbb{R}\right)\right\}$ over $B$.

- The closed 1-form $\omega_{b}$ is Morse for $b$ in the complement of a codimension one subvariety of $B$. 
In particular, admissibility implies that the groups $K_{b}:=\operatorname{Ker}\left(\omega_{b}\right) \subset H_{1}\left(Z_{b}\right)$ and $\Gamma_{b}:=H_{1}\left(Z_{b}\right) / K_{b}$, and the Novikov rings $\Lambda_{b}=\operatorname{Nov}\left(\Gamma_{b},-\omega_{b} ; \mathbb{Z}\right)$, comprise local coefficient systems on $B$, which we denote by $K, \Gamma$, and $\Lambda$ respectively.

Now suppose $\gamma:[0,1] \rightarrow B$ is a smooth path and $x_{0} \in Z_{\gamma(0)}$ and $x_{1} \in Z_{\gamma(1)}$ are reference points. Trivializing $\gamma^{*} Z$ and applying Lemma 9.6 shows that a relative homology class $\chi \in H_{1}\left(\gamma^{*} Z,\left(0, x_{0}\right),\left(1, x_{1}\right)\right) / K$ determines a continuation isomorphism

$$
\Phi(\gamma, \chi): H_{*}^{\mathrm{Nov}}\left(\omega_{\gamma}(0), x_{0}\right) \stackrel{\simeq}{\longrightarrow} H_{*}^{\mathrm{Nov}}\left(\omega_{\gamma(1)}, x_{1}\right) .
$$

This isomorphism is invariant under homotopy of $\gamma$ rel endpoints (together with appropriate replacement of $\chi$ ), equals the identity when $\gamma$ is constant and $\chi=0$, and composes for composable paths.

We will use the above continuation isomorphisms to assemble the Novikov homologies of the $\omega_{b}$ 's into a local coefficient system on $B$. This requires the following additional structure.

Definition 9.8 A family of reference points consists of:

(a) For each $b \in B$, a reference point $x_{b} \in Z_{b}$. (The point $x_{b}$ is not required to depend continuously on $b$.)

(b) For each path $\gamma:[0,1] \rightarrow B$, a relative homology class

$$
\chi_{\gamma} \in H_{1}\left(\gamma^{*} Z,\left(0, x_{\gamma(0)}\right),\left(1, x_{\gamma(1)}\right)\right) / K .
$$

We impose the following conditions on the relative homology classes $\chi_{\gamma}$ :

(i) If $\gamma$ is a constant path mapping to $b \in B$, then $\chi_{\gamma}=\left[[0,1] \times\left\{x_{b}\right\}\right]$.

(ii) If $\gamma_{0}$ and $\gamma_{1}$ are homotopic rel endpoints, then the isomorphism

$$
H_{1}\left(\gamma_{0}^{*} Z,\left(0, x_{\gamma_{0}(0)}\right),\left(1, x_{\gamma_{0}(1)}\right)\right) / K \simeq H_{1}\left(\gamma_{1}^{*} Z,\left(0, x_{\gamma_{1}(0)}\right),\left(1, x_{\gamma_{1}(1)}\right)\right) / K
$$

induced by the homotopy sends $\chi_{\gamma_{0}} \mapsto \chi_{\gamma_{1}}$.

(iii) If $\gamma_{1}$ and $\gamma_{2}$ are composable paths then $\chi_{\gamma_{1} \gamma_{2}}=\chi_{\gamma_{1}}+\chi_{\gamma_{2}}$.

Definition 9.9 An isomorphism between two families of reference points $\left\{x_{b}, \chi_{\gamma}\right\}$ and $\left\{x_{b}^{\prime}, \chi_{\gamma}^{\prime}\right\}$ consists of an element $\rho_{b} \in H_{1}\left(Z_{b}, x_{b}, x_{b}^{\prime}\right) / K_{b}$ for each $b \in B$, such that for every path $\gamma:[0,1] \rightarrow B$, we have

$$
\chi_{\gamma}+\rho_{\gamma(1)}=\rho_{\gamma(0)}+\chi_{\gamma}^{\prime} \in H_{1}\left(\gamma^{*} Z,\left(0, x_{\gamma(0)}\right),\left(1, x_{\gamma(1)}^{\prime}\right)\right) / K .
$$

For example, it follows immediately from the definition that if $R$ is a family of reference points, then $\operatorname{Aut}(R)=H^{0}(B ; \Gamma)$ 
Proposition 9.10 Let $(Z, \omega)$ be an admissible family and let $R=\left\{x_{b}, \chi_{\gamma}\right\}$ be a family of reference points. Then:

(a) The continuation isomorphisms $\Phi\left(\gamma, \chi_{\gamma}\right)$ assemble the Novikov homologies $H_{*}^{\text {Nov }}\left(\omega_{b}, x_{b}\right)$ into a local coefficient system $\mathcal{F}_{*}(Z, \omega, R)$, which is a $\mathbb{Z}$-graded module over the local coefficient system $\Lambda$.

(b) Given another family of reference points $R^{\prime}$, an isomorphism $R \simeq R^{\prime}$ induces an isomorphism $\mathcal{F}_{*}(Z, \omega, R) \simeq \mathcal{F}_{*}\left(Z, \omega, R^{\prime}\right)$.

(c) An automorphism $A \in \operatorname{Aut}(R)=H^{0}(B ; \Gamma)$ acts on $\mathcal{F}_{*}(Z, \omega, R)$ by multiplication by $e^{A} \in \Lambda$.

Proof This is an immediate formal consequence of the preceding definitions and the homotopy properties of the continuation isomorphisms $\Phi\left(\gamma, \chi_{\gamma}\right)$.

We turn now to the question of the existence and classification of families of reference points. Let $\mathcal{R}$ denote the set of isomorphism classes of families of reference points. The primary obstruction to the existence of a section of $Z \rightarrow B$ determines a cohomology class

$$
\mathfrak{o} \in H^{2}\left(B ;\left\{H_{1}\left(Z_{b}\right)\right\}\right) .
$$

A straightforward obstruction theory argument then proves the following:

Proposition 9.11 (a) $\mathcal{R} \neq \varnothing$ if and only if $\mathfrak{o} \equiv 0 \in H^{2}(B ; \Gamma)$.

(b) $\mathcal{R}$, if nonempty, is an affine space over $H^{1}(B ; \Gamma)$.

Example 9.12 If $B=S^{1}$, then continuation around $S^{1}$ defines a monodromy isomorphism $H_{*}^{\text {Nov }}\left(\omega_{0}, x_{0}\right) \stackrel{\simeq}{\longrightarrow} H_{*}^{\text {Nov }}\left(\omega_{0}, x_{0}\right)$, which is a priori defined only up to multiplication by $e^{A}$ for $A \in \Gamma$, and becomes well defined once an element of $\mathcal{R}$ is chosen. Seidel [39] makes a corresponding choice to define the monodromy in symplectic Floer homology for a loop of Hamiltonian symplectomorphisms.

The following proposition guarantees the existence of families of reference points in many cases of interest.

Proposition 9.13 Let $b_{0} \in B$ and suppose that $H_{*}^{\mathrm{Nov}}\left(\omega_{b_{0}}, x_{b_{0}}\right) \neq 0$. Then the $o b$ struction class $\mathfrak{o}$ annihilates $\pi_{2}\left(B, b_{0}\right)$ under the evaluation paring

$$
H^{2}(B ; \Gamma) \otimes \pi_{2}\left(B, b_{0}\right) \longrightarrow \Gamma_{b_{0}}
$$


Proof Fix a reference point $x_{0} \in Z_{b_{0}}$. Consider an element of $\pi_{2}\left(B, b_{0}\right)$, represented by a homotopy $H$ of loops based at $b_{0}$, starting and ending at the constant path. Let

$$
A:=\langle\mathfrak{o}, H\rangle \in H_{1}\left(Z_{b_{0}}\right) .
$$

To describe $A$ more explicitly, note that the homotopy $H$ induces an automorphism of $H_{1}\left([0,1] \times Z_{b_{0}},\left(0, x_{0}\right),\left(1, x_{0}\right)\right)$, which is just translation by the homology class $A$. We need to show that $A \in K_{b_{0}}$, ie $\omega_{b_{0}}(A)=0$.

Choose a metric $g$ on $Z_{b_{0}}$ to define the Novikov complex $C_{*}^{\text {Nov }}\left(\omega_{b_{0}}, g, x_{0}\right)$. For any class

$$
\chi \in H_{1}\left([0,1] \times Z_{b_{0}},\left(0, x_{0}\right),\left(1, x_{0}\right)\right)=H_{1}\left(Z_{b_{0}}\right),
$$

continuation along the constant path at $b_{0}$ defines a continuation chain map

$$
\Phi(\chi): C_{*}^{\mathrm{Nov}}\left(\omega_{b_{0}}, g, x_{0}\right) \longrightarrow C_{*}^{\mathrm{Nov}}\left(\omega_{b_{0}}, g, x_{0}\right),
$$

which is just multiplication by $e^{\chi}$. Fix $\chi=0$.

The homotopy $H$, together with a generic 2 -parameter family of metrics, defines a chain homotopy of continuation maps

$$
L: C_{*}^{\mathrm{Nov}}\left(\omega_{b_{0}}, g, x_{0}\right) \longrightarrow C_{*+1}^{\mathrm{Nov}}\left(\omega_{b_{0}}, g, x_{0}\right)
$$

satisfying

$$
\begin{aligned}
\partial L+L \partial & =\Phi(0)-\Phi(A) \\
& =1-e^{A} .
\end{aligned}
$$

If $\omega_{b_{0}}(A) \neq 0$, then the right hand side of (9-1) is a unit in the Novikov ring $\Lambda_{b_{0}}$, because without loss of generality $\omega_{b_{0}}(A)<0$, and then

$$
\left(1-e^{A}\right)^{-1}=1+e^{A}+e^{2 A}+\cdots
$$

satisfies the finiteness criterion for membership in $\Lambda_{b_{0}}$. Since multiplication by a unit is chain homotopic to zero, it follows that $H_{*}^{\mathrm{Nov}}\left(\omega_{b_{0}}, x_{0}\right)=0$, contradicting the hypothesis of the proposition.

For example, combining the above with the Hurewicz theorem, we obtain:

Corollary 9.14 If $B$ is connected and simply connected and $H_{*}^{\mathrm{Nov}}\left(\omega_{b}, x_{b}\right) \neq 0$, then there exists a family of reference points, which is unique up to isomorphism. 


\subsection{Novikov homology of families}

We are now prepared to state a version of the Main Principle for Novikov homology.

Let $\pi: Z \rightarrow B$ be a smooth fiber bundle whose fibers are closed manifolds, let $\left\{\omega_{b} \mid\right.$ $b \in B\}$ be an admissible family of closed 1 -forms on the fibers, and let $R=\left\{x_{b}, \chi_{\gamma}\right\}$ be a family of reference points. We define a filtered chain complex $\mathfrak{C}_{*}(Z, \omega, R)$, generalizing the construction in Section 3.1, as follows.

Let $\mathcal{A}_{i}$ denote the set of nondegenerate pairs $(\sigma, g)$, where $\sigma:[-1,1]^{i} \rightarrow B$ is a smooth $i$-cube and $g$ is a fiberwise metric on $\sigma^{*} Z$, that are admissible as in Definition 3.3. Define

$$
\mathfrak{C}_{i, j}:=\bigoplus_{(\sigma, g) \in \mathcal{A}_{i}} C_{j}^{\mathrm{Nov}}\left(\omega_{\sigma(0)}, g_{0}, x_{\sigma(0)}\right) .
$$

For $0 \leq k \leq i$, define

$$
\delta_{k}: \mathfrak{C}_{i, j} \longrightarrow \mathfrak{C}_{i-k, j+k-1}
$$

as follows. Let $(\sigma, g) \in \mathcal{A}_{i}$ and let $\tilde{p}=(p, \eta)$ be an anchored critical point of $\omega_{\sigma(0)}$. In the notation of (3-6), define

$$
\delta_{k}(\sigma, g, \widetilde{p}):=\sum_{\substack{\sigma^{\prime} \in F_{k}(\sigma) \\ q \in \operatorname{Crit}_{j+k+1}\left(\sigma^{\prime}(0)\right) \\ \mu \in H_{1}\left(\sigma^{*} Z, p, q\right)}} \# \mathcal{M}(p, q, \mu) \cdot\left(\sigma^{\prime}, g_{\sigma^{\prime}},\left(q, \eta+\chi_{\sigma, \sigma^{\prime}}-\mu\right)\right)
$$

Here $\mathcal{M}(p, q, \mu)$ denotes the moduli space of flow lines of the vector field (3-5) from $p$ to $q$ in the relative homology class $\mu$. Also $\chi_{\sigma, \sigma^{\prime}}$ denotes $\chi_{\sigma \circ \gamma}$, where $\gamma$ is any path in $[-1,1]^{i}$ from 0 to the center of the face $\sigma^{\prime}$. Finally, any summands in which the pair $\left(\sigma^{\prime}, g_{\sigma^{\prime}}\right)$ is degenerate are implicitly discarded from the above sum.

We now define $\mathfrak{C}_{*}:=\bigoplus_{i+j=*} \mathfrak{C}_{i, j}$. This has a filtration given by $i$ as in (3-11), and it is a module over $H^{0}(B ; \Lambda)$. We define the differential $\delta:=\sum_{k} \delta_{k}: \mathfrak{C}_{*} \rightarrow \mathfrak{C}_{*-1}$. The usual arguments show that $\delta$ is well defined and $\delta^{2}=0$. The filtered chain complex $\mathfrak{C}_{*}(Z, \omega, R)$ has a homology, the family Novikov homology $H_{*}(Z, \omega, R)$, and a spectral sequence $E_{*, *}^{*}(Z, \omega, R)$ which converges to it. These satisfy straightforward analogues of the properties in the Main Principle. We state the first three properties and omit the rest:

Proposition 9.15 (a) $E_{i, j}^{2}(Z, \omega, R)=H_{i}\left(B ; \mathcal{F}_{j}(Z, \omega, R)\right)$.

(b) (Homotopy invariance) An isomorphism of families of reference points $R \simeq R^{\prime}$ induces an isomorphism of filtered chain complexes

$$
\mathfrak{C}_{*}(Z, \omega, R) \simeq \mathfrak{C}_{*}\left(Z, \omega, R^{\prime}\right) .
$$


More generally, let $\pi: Z \rightarrow[0,1] \times B$ be a smooth fiber bundle whose fibers are closed manifolds. Let $\left\{\omega_{(t, b)} \mid t \in[0,1], b \in B\right\}$ be a family of closed 1forms on the fibers, whose cohomology classes are locally constant, such that the families $\left\{\omega_{(0, b)} \mid b \in B\right\}$ and $\left\{\omega_{(1, b)} \mid b \in B\right\}$ are admissible. Let $R$ be a family of reference points over $[0,1] \times B$. Then there is a filtered chain homotopy equivalence

$$
\Phi: \mathfrak{C}_{*}\left(\left.(Z, \omega, R)\right|_{\{0\} \times B}\right) \longrightarrow \mathfrak{C}_{*}\left((Z, \omega, R)_{\{1\} \times B}\right) .
$$

This satisfies the homotopy properties of Proposition 8.6(i)-(iii). The induced map

$$
E_{i, j}^{2}\left(\left.(Z, \omega, R)\right|_{\{0\} \times B}\right) \longrightarrow E_{i, j}^{2}\left(\left.(Z, \omega, R)\right|_{\{1\} \times B}\right)
$$

is the canonical isomorphism determined by continuation.

(c) (Naturality) If $\phi: B^{\prime} \rightarrow B$ is a generic smooth map, so that the pullback $\left(\phi^{*} Z, \phi^{*} \omega\right)$ is admissible, then there is a canonical filtered chain map

$$
\phi_{*}: \mathfrak{C}_{*}\left(\phi^{*} Z, \phi^{*} \omega, \phi^{*} R\right) \longrightarrow \mathfrak{C}_{*}(Z, \omega, R) .
$$

This map is functorial, and homotopy invariant up to filtered chain homotopy, as in Proposition 8.7. On the $E^{2}$ terms of the spectral sequences, $\phi_{*}$ induces the homology pushforward

$$
\phi_{*}: H_{*}\left(B^{\prime} ; \mathcal{F}_{*}\left(\phi^{*} Z, \phi^{*} \omega, \phi^{*} R\right)\right) \longrightarrow H_{*}\left(B ; \mathcal{F}_{*}(Z, \omega, R)\right) .
$$

The proof is the same as before, except that one needs additional notation to keep track of the relative homology classes of flow lines, and one needs to see that everything is well-defined over the Novikov ring, using a version of the energy bound (3-10).

One can also define a simpler version of the above filtered chain complex using Morse homology on the base as in Section 6.1. To do so, fix Morse data $\left(f^{B}, g^{B}\right)$ on $B$ and a generic fiberwise metric $g^{Z}$ as in Section 6.1. Define a filtered chain complex $\mathcal{C}_{*}\left(Z, \omega, R, g^{Z}, f^{B}, g^{B}\right)$ as follows. First define

$$
\mathcal{C}_{i, j}:=\bigoplus_{b \in \operatorname{Crit}_{i}\left(f^{B}\right)} C_{j}^{\mathrm{Nov}}\left(\omega_{b}, g_{b}^{Z}, x_{b}\right)
$$

For $k \geq 0$ define $\delta_{k}: \mathcal{C}_{i, j} \rightarrow \mathcal{C}_{i-k, j+k-1}$ as follows: If $b \in \operatorname{Crit}_{i}\left(f^{\boldsymbol{B}}\right)$ and $\tilde{p}=(p, \eta)$ is an anchored index $j$ critical point of $\omega_{b}$, then

$$
\delta_{k}(b, \tilde{p}):=\sum_{\substack{b^{\prime} \in \operatorname{Crit}_{i-k}\left(f^{B}\right) \\ p^{\prime} \in \operatorname{Crit}_{j+k-1}\left(\omega_{b^{\prime}}\right)}} \sum_{u \in \mathcal{M}\left((b, p),\left(b^{\prime}, p^{\prime}\right)\right)} \varepsilon(u)\left(b^{\prime},\left(p^{\prime}, \eta+\chi_{\pi \circ u}-[u]\right)\right) .
$$


Here $\mathcal{M}\left((b, p),\left(b^{\prime}, p^{\prime}\right)\right)$ denotes the moduli space of flow lines of the vector field (6-1), and $\varepsilon(u) \in\{ \pm 1\}$ denotes the sign associated to $u$. We then define $\mathcal{C}_{*}:=\bigoplus_{i+j=*} \mathcal{C}_{i, j}$ and $\delta:=\sum_{k} \delta_{k}$. The usual arguments show that $\delta$ is well defined and $\delta^{2}=0$. Furthermore, the filtered chain complex $\mathcal{C}_{*}$ has the same spectral sequence and the same homology (and conjecturally the same filtered chain homotopy type) as $\mathfrak{C}_{*}$.

\section{Genericity and transversality}

To finish up, we now prove Proposition 3.4. The proof uses the Sard-Smale theorem, and boils down to checking that a certain operator is surjective and another operator is Fredholm. As we will see below, the proofs of the surjectivity and Fredholm properties for the fiberwise statement of Proposition 3.4 reduce to the corresponding calculations for proving that the gradient of a single Morse function with respect to a generic metric is Morse-Smale (together with the fact that the vector field $W_{i}$ is Morse-Smale).

Proof of Proposition 3.4 Fix a positive integer $r>\operatorname{dim}(Z)$ and let $\operatorname{Met}^{r}$ denote the space of $C^{r}$ fiberwise metrics on $\sigma^{*} Z$ extending $g_{0}$. We will prove that the vector field $V$ on $\sigma^{*} Z$ is Morse-Smale for a Baire set of $g \in \mathrm{Met}^{r}$. (One can then pass from $C^{r}$ to $C^{\infty}$ as in [29].)

Note that the critical points of $V$ do not depend on the choice of metric. Fix distinct critical points $p$ and $q$. We need to show that for generic $g$, the unstable manifold of $p$ is transverse to the stable manifold of $q$. Since the codimension one faces with the metric $g_{0}$ are admissible, we may assume without loss of generality that $p$ is over the center of the cube.

We begin by setting up a "universal moduli space" of flow lines of $V$ from $p$ to $q$ as the zero set of a section of a vector bundle. Define:

$$
\mathcal{P}:=\left\{\begin{array}{l|l}
(g, \gamma, \zeta) & \begin{array}{l}
g \in \mathrm{Met}^{r} \\
\gamma \in L_{1}^{2}\left(\mathbb{R},[-1,1]^{i}\right) \\
\zeta \in L_{1}^{2}(\mathbb{R}, Z) \\
\pi \circ \zeta=\sigma \circ \gamma \\
\lim _{s \rightarrow-\infty} \zeta(s)=p, \lim _{s \rightarrow+\infty} \zeta(s)=q
\end{array}
\end{array}\right\}
$$

Note that a pair $(\gamma, \zeta)$ satisfying $\pi \circ \zeta=\sigma \circ \gamma$ is equivalent to a path in $\sigma^{*} Z$. The spaces $L_{1}^{2}$ above are defined using fixed coordinate charts in $Z$ around the critical 
points $p$ and $q$. Now $\mathcal{P}$ is a Banach manifold whose tangent space is:

$$
T_{(g, \gamma, \zeta)} \mathcal{P}=\left\{\begin{array}{l|l}
(\dot{g}, \dot{\gamma}, \dot{\zeta}) & \begin{array}{l}
\dot{g} \in T_{g} \mathrm{Met}^{r} \\
\dot{\gamma} \in L_{1}^{2}\left(\mathbb{R}, \mathbb{R}^{i}\right) \\
\dot{\zeta} \in L_{1}^{2}\left(\zeta^{*} T Z\right) \\
\pi_{*} \dot{\zeta}=\sigma_{*} \dot{\gamma}
\end{array}
\end{array}\right\}
$$

Let $T^{\text {vert }} Z$ denote the vertical tangent bundle to $Z$. Define a Banach space bundle $\mathcal{E}$ on $\mathcal{P}$ by

$$
\mathcal{E}_{(g, \gamma, \zeta)}:=L^{2}\left(\mathbb{R}, \mathbb{R}^{i}\right) \oplus L^{2}\left(\zeta^{*} T^{\mathrm{vert}} Z\right) .
$$

This is a $C^{\infty}$ Banach space bundle if we use some fixed smooth metric on $Z$ to define the coordinate charts. Define a $C^{r}$ section $\psi$ of $\mathcal{E}$ by

$$
\psi(g, \gamma, \zeta):=\left(\gamma^{\prime}-W_{i}(\gamma), \nabla_{\sigma_{*} \gamma^{\prime}}(\zeta)-\xi(\zeta)\right)
$$

Here $\xi$ denotes the fiberwise negative gradient as before. By definition, $(g, \gamma, \zeta)$ is a zero of $\psi$ if and only if $(\gamma, \zeta)$ is a flow line of the vector field $V$ for the pair $(\sigma, g)$. Let $\rho: \mathcal{P} \rightarrow \operatorname{Met}^{r}$ denote the projection.

Lemma 10.1 (a) The zero locus $\psi^{-1}(0)$ is a $C^{r}$ submanifold of $\mathcal{P}$.

(b) For $x \in \psi^{-1}(0)$, the restricted differential

$$
\mathcal{D}:=d \psi: T_{x} \mathcal{P}_{\rho(x)} \longrightarrow \mathcal{E}_{x}
$$

is Fredholm.

Proof To prove both (a) and (b), let $(g, \gamma, \zeta) \in \psi^{-1}(0)$ be given.

(a) By the implicit function theorem, it is enough to show that the differential

$$
d \psi: T_{(g, \gamma, \zeta)} \mathcal{P} \longrightarrow \mathcal{E}_{(g, \gamma, \zeta)}
$$

is surjective. The connection $\nabla$ on $Z$ allows us to identify

$$
T_{(g, \gamma, \zeta)} \mathcal{P}=T_{g} \operatorname{Met}^{r} \oplus L_{1}^{2}\left(\mathbb{R}, \mathbb{R}^{i}\right) \oplus L_{1}^{2}\left(\zeta^{*} T^{\mathrm{vert}} Z\right)
$$

Using this identification, we compute that

$\left.(10-1) d \psi\left(\dot{g}, \dot{\gamma}, \dot{\zeta}^{\text {vert }}\right)=\left(\dot{\gamma}^{\prime}-d W_{i}(\dot{\gamma}), \nabla_{\sigma_{*} \gamma^{\prime}} \dot{\zeta}^{\text {vert }}+F_{1}(\dot{\gamma})+F_{2}\left(\dot{\zeta}^{\text {vert }}\right)-d \xi(\dot{g})\right)\right)$.

Here $F_{1}$ and $F_{2}$ are zeroth order operators whose precise form is not relevant for this argument, and $d \xi(\dot{g})$ denotes the derivative of $\xi$ with respect to $\dot{g}$ at $\zeta$. 
Suppose that $\left(\eta_{0}, \eta_{1}\right) \in \mathcal{E}_{(g, \gamma, \xi)}$ is orthogonal to the image of $d \psi$. Then $\eta_{0} \equiv 0$, because the vector field $W_{i}$ is Morse-Smale. Furthermore,

$$
\int_{-\infty}^{\infty}\left\langle d \xi(\dot{g}), \eta_{1}\right\rangle((\gamma, \zeta)(s)) d s=0
$$

for all $\dot{g} \in T_{g} \operatorname{Met}^{r}$. This implies that $\eta_{1} \equiv 0$, because the flow line $(\gamma, \zeta): \mathbb{R} \rightarrow \sigma^{*} Z$ is injective, and $d \xi$ is surjective at each (noncritical) point: given a nonzero covector, one can move its metric dual in any direction by deforming the metric.

(b) By Equation (10-1), the restricted differential $\mathcal{D}$ fits into a commutative diagram with short exact rows,

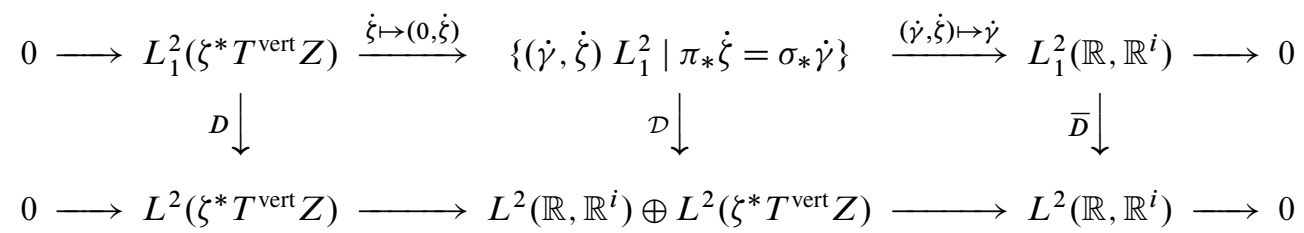

where

$$
\begin{aligned}
D\left(\dot{\zeta}^{\mathrm{vert}}\right) & :=\nabla_{\sigma_{*} \gamma^{\prime}} \dot{\zeta}^{\mathrm{vert}}+F_{2}\left(\dot{\zeta}^{\mathrm{vert}}\right), \\
\bar{D}(\dot{\gamma}) & :=\dot{\gamma}^{\prime}-d W_{i}(\dot{\gamma}) .
\end{aligned}
$$

It is standard that the operators $D$ and $\bar{D}$ are Fredholm; cf [34]. Since $D$ and $\bar{D}$ have finite dimensional kernel and cokernel, it follows by the snake lemma that $\mathcal{D}$ does as well. Thus $\mathcal{D}$ is Fredholm $($ and $\operatorname{ind}(\mathcal{D})=\operatorname{ind}(D)+\operatorname{ind}(\bar{D}))$.

In conclusion, we know that $\mathcal{E} \rightarrow \mathcal{P}$ is a separable Banach space bundle, $\rho: \mathcal{P} \rightarrow \operatorname{Met}^{r}$ is a Banach manifold fiber bundle, $\psi: \mathcal{P} \rightarrow \mathcal{E}$ is a $C^{r}$ section, and Lemma 10.1 holds. It follows from the Sard-Smale theorem (cf [29]) that a generic metric $g \in \mathrm{Met}^{r}$ is a regular value of the projection $\rho: \psi^{-1}(0) \rightarrow \mathrm{Met}^{r}$. It is a standard matter to show that $g$ is a regular value of $\rho: \psi^{-1}(0) \rightarrow \operatorname{Met}^{r}$ if and only if the unstable manifold of $p$ for $V$ is transverse to the stable manifold of $q$. This completes the proof of Proposition 3.4.

\section{References}

[1] D M Austin, P J Braam, Morse-Bott theory and equivariant cohomology, from: "The Floer memorial volume”, Progr. Math. 133, Birkhäuser, Basel (1995) 123-183 MR1362827

[2] J-F Barraud, O Cornea, Lagrangian intersections and the Serre spectral sequence, Annals of Math. 166 (2007) 657-722 
[3] P Biran, Lagrangian barriers and symplectic embeddings, Geom. Funct. Anal. 11 (2001) 407-464 MR1844078

[4] J-M Bismut, S Goette, Families torsion and Morse functions, Astérisque (2001) x+293 MR1867006

[5] R Bott, An application of the Morse theory to the topology of Lie-groups, Bull. Soc. Math. France 84 (1956) 251-281 MR0087035

[6] R Bott, Morse theory indomitable, Inst. Hautes Études Sci. Publ. Math. (1988) 99-114 (1989) MR1001450

[7] F Bourgeois, Contact homology and homotopy groups of the space of contact structures, Math. Res. Lett. 13 (2006) 71-85 MR2200047

[8] Y Chekanov, Differential algebra of Legendrian links, Invent. Math. 150 (2002) 441483 MR1946550

[9] R Cohen, J Jones, G Segal, Morse theory and classifying spaces, Warwick Univ. preprint (1995) Available at http://math.stanford.edu/ ralph/morse.ps

[10] O Cornea, A Ranicki, Rigidity and gluing for Morse and Novikov complexes, J. Eur. Math. Soc. (JEMS) 5 (2003) 343-394 MR2017851

[11] T Ekholm, T Kálmán, Isotopies of Legendrian 1-knots and Legendrian 2-tori arXiv: 0710.4382

[12] Y Eliashberg, A Givental, H Hofer, Introduction to symplectic field theory, Geom. Funct. Anal. (2000) 560-673 MR1826267GAFA 2000 (Tel Aviv, 1999)

[13] J B Etnyre, L L Ng, J M Sabloff, Invariants of Legendrian knots and coherent orientations, J. Symplectic Geom. 1 (2002) 321-367 MR1959585

[14] A Floer, Morse theory for Lagrangian intersections, J. Differential Geom. 28 (1988) 513-547 MR965228

[15] K Fukaya, Floer homology of connected sum of homology 3-spheres, Topology 35 (1996) 89-136 MR1367277

[16] K Fukaya, Floer homology for families - report of a project in progress, preprint (2001) MR1905880 Available at http:/www.math.kyoto-u.ac.jp/ fukaya/ familyy.pdf

[17] K Fukaya, P Seidel, I Smith, The symplectic geometry of cotangent bundles from a categorical viewpoint arXiv:0705.3450

[18] H Geiges, J Gonzalo Perez, On the topology of the space of contact structures on torus bundles, Bull. London Math. Soc. 36 (2004) 640-646 MR2070440

[19] A Hatcher, Algebraic topology, Cambridge University Press, Cambridge (2002) MR1867354

[20] H Hofer, D A Salamon, Floer homology and Novikov rings, from: "The Floer memorial volume”, Progr. Math. 133, Birkhäuser, Basel (1995) 483-524 MR1362838 
[21] M Hutchings, Floer homology of families II: symplectomorphisms, in preparation

[22] M Hutchings, Reidemeister torsion in generalized Morse theory, Forum Math. 14 (2002) 209-244 MR1880912

[23] M Hutchings, Y-J Lee, Circle-valued Morse theory, Reidemeister torsion, and SeibergWitten invariants of 3-manifolds, Topology 38 (1999) 861-888 MR1679802

[24] T Kálmán, Contact homology and one parameter families of Legendrian knots, Geom. Topol. 9 (2005) 2013-2078 MR2209366

[25] P Kronheimer, T Mrowka, Monopoles and three-manifolds, New Mathematical Monographs 10, Cambridge University Press (2007)

[26] P Kronheimer, T Mrowka, P Ozsváth, Z Szabó, Monopoles and lens space surgeries, Ann. of Math. (2) 165 (2007) 457-546 MR2299739

[27] J Latschev, A generalization of the Morse complex, PhD thesis, SUNY Stony Brook (1998)

[28] F Laudenbach, On the Thom-Smale complex, Astérisque (1992) 219-233

[29] D McDuff, D Salamon, J-holomorphic curves and quantum cohomology, University Lecture Series 6, Amer. Math. Soc. (1994) MR1286255

[30] A Oancea, Fibered symplectic cohomology and the Leray-Serre spectral sequence arXiv:math/0503193

[31] A Oancea, La suite spectrale de Leray-Serre en homologie de Floer des variétés symplectiques compactes à bord de type contact, $\mathrm{PhD}$ thesis, Université Paris Sud, Orsay (2003)

[32] P Ozsváth, Z Szabó, Holomorphic disks and topological invariants for closed threemanifolds, Ann. of Math. (2) 159 (2004) 1027-1158 MR2113019

[33] M Poźniak, Floer homology, Novikov rings and clean intersections, from: "Northern California Symplectic Geometry Seminar”, Amer. Math. Soc. Transl. Ser. 2 196, Amer. Math. Soc. (1999) 119-181 MR1736217

[34] J Robbin, D Salamon, The spectral flow and the Maslov index, Bull. London Math. Soc. 27 (1995) 1-33 MR1331677

[35] D Salamon, Lectures on Floer homology, from: "Symplectic geometry and topology (Park City, UT, 1997)”, (Y Eliashberg, editor), IAS/Park City Math. Ser. 7, Amer. Math. Soc. (1999) 143-229 MR1702944

[36] Y Savelyev, Quantum characteristic classes and the Hofer metric arXiv:0709.4510

[37] M Schwarz, Morse homology, Progress in Mathematics 111, Birkhäuser Verlag, Basel (1993) MR1239174

[38] P Seidel, Floer homology and the symplectic isotopy problem, $\mathrm{PhD}$ thesis, Oxford University (1997) 
[39] P Seidel, $\pi_{1}$ of symplectic automorphism groups and invertibles in quantum homology rings, Geom. Funct. Anal. 7 (1997) 1046-1095 MR1487754

[40] C Viterbo, Functors and computations in Floer homology with applications. I, Geom. Funct. Anal. 9 (1999) 985-1033 MR1726235

[41] E Witten, Supersymmetry and Morse theory, J. Differential Geom. 17 (1982) 661-692 (1983) MR683171

Mathematics Department, 970 Evans Hall, University of California

Berkeley, CA 94720

hutching@math.berkeley.edu

http://math. berkeley.edu/ hutching

Received: 8 November 2007

Algebraic 83 Geometric Topology, Volume 8 (2008) 